\title{
Direct Scaling Analysis of Fermionic Multiparticle Correlated Anderson Models with Infinite-Range Interaction
}

\author{
Victor Chulaevsky \\ Département de Mathématiques, Université de Reims, Moulin de la Housse, BP 1039, 51687 Reims Cedex 2, France \\ Correspondence should be addressed to Victor Chulaevsky; plinius@free.fr
}

Received 26 August 2015; Accepted 13 December 2015

Academic Editor: Hagen Neidhardt

Copyright ( 2016 Victor Chulaevsky. This is an open access article distributed under the Creative Commons Attribution License, which permits unrestricted use, distribution, and reproduction in any medium, provided the original work is properly cited.

\begin{abstract}
We adapt the method of direct scaling analysis developed earlier for single-particle Anderson models, to the fermionic multiparticle models with finite or infinite interaction on graphs. Combined with a recent eigenvalue concentration bound for multiparticle systems, the new method leads to a simpler proof of the multiparticle dynamical localization with optimal decay bounds in a natural distance in the multiparticle configuration space, for a large class of strongly mixing random external potentials. Earlier results required the random potential to be IID.
\end{abstract}

\section{Introduction}

1.1. The Model: A Brief Historical Overview. Analysis of localization phenomena in multiparticle quantum systems with nontrivial interaction in a random environment is a relatively new direction in the Anderson localization theory, where during almost half a century, since the seminal paper by Anderson [1], most efforts were concentrated on the study of disordered systems in the single-particle approximation, that is, without interparticle interaction. While in numerous physical models such an approximation seems fairly reasonable, it was pointed out already in the first works by Anderson that the multiparticle models presented a real challenge.

The number of results on multiparticle localization obtained in the physical and mathematical communities still remains rather limited. We do not review here results obtained by physicists (cf. $[2,3])$, based on the methods of theoretical physics and considered as firmly established by the physical community. The rigorous mathematical results on multiparticle localization obtained so far (cf., e.g., [4-7]) apply to $N$-particle systems with arbitrary, but fixed $N>1$, and the range of parameters (such as the amplitude of the disorder and/or the proximity to the edge(s) of the spectrum) is rapidly degrading as $N \rightarrow \infty$.
In this paper, we study spectral properties of the multiparticle random Hamiltonians of the form

$$
\mathbf{H}(\omega)=\mathbf{H}_{0}+g \mathbf{V}(\omega)+\mathbf{U}, \quad g \in \mathbb{R},
$$

describing $N>1$ fermionic particles in the configuration space $\mathscr{Z}$ assumed to be a finite or countable graph. For clarity, we present first our method in the particular case where $\mathscr{Z}=\mathbb{Z}^{1}$ and then describe how to adapt the arguments to more general graphs $\mathscr{Z}$ with polynomial growth of balls (cf. Section 8). In (1), $\mathbf{H}_{0}$ is a finite-difference operator representing the kinetic energy, for example, the nearestneighbor discrete negative Laplacian $(-\boldsymbol{\Delta}), \mathrm{U}$ is the operator of multiplication by the interaction potential $\mathbf{x} \mapsto \mathbf{U}(\mathbf{x})$, and $\mathbf{V}(\omega)$ is the operator of multiplication by the random function

$$
\mathbf{x} \longmapsto \mathbf{V}(\mathbf{x} ; \omega)=V\left(x_{1} ; \omega\right)+\cdots+V\left(x_{N} ; \omega\right),
$$

where $V: \mathscr{Z} \times \Omega \rightarrow \mathbb{R}$ is a random field relative to some probability space $(\Omega, \mathscr{F}, \mathbb{P}) . g>0$ is a parameter measuring the amplitude of disorder. (All notations are explained in detail in Section 2.)

We consider only the restriction of $\mathbf{H}(\omega)$ to the fermionic subspace. The bosonic subspace can be treated essentially in the same way. 
1.2. Motivation for This Work. The two most popular methods of the rigorous Anderson localization theory, the Multiscale Analysis developed in [8-11] and the Fractional Moments Method developed in $[12,13]$, start with the analysis of the Green functions (GFs) of a given random operator, and then one has to translate the upper bounds on the GFs obtained for a fixed energy (cf. $[8,11-13]$ ) or in an energy interval (cf. $[8,10])$ into the language of the eigenfunctions (EFs) and eigenfunction correlators (EFCs). In a prior work [14] we proposed an alternative approach, called the Direct Scaling Analysis of eigenfunctions, which focuses essentially on the eigenfunctions in finite domains of the configuration space, from the very beginning. It keeps some important advantages of the MSA, namely, a greater tolerance than in FMM to lower regularity of the disorder, and is close in spirit to the KAM (Kolmogorov-Arnold-Moser) approach, which often requires a tedious inductive scheme. In fact, work [14] has been inspired by the KAM approach to quasi-periodic Hamiltonians proposed earlier by Sinai [15]. Compared to the KAM method, the DSA has a simpler structure of the scale induction, similar to that of the MSA, although it also inherits somewhat weaker probabilistic bounds typical of the MSA.

Furthermore, as was said, there are two kinds of the MSA: with a fixed energy (FEMSA) and with a variable energy (VEMSA); the latter is often referenced to as the energyinterval MSA, for it treats the decay properties of the Green functions in an entire interval of energies at once. The FEMSA is slightly simpler than VEMSA in the single-particle case, but in the realm of multiparticle random Hamiltonians with a nontrivial interaction, the variable-energy variant of the (multiparticle) MSA is considerably more complex. Some of the components of the VEMPMSA are simply eliminated with the (MP)DSA approach, and the counterparts of some other components become quite elementary. A good example of such kind is Lemma 21 (see Section 4.3), the proof of which is very simple and fits in a few lines, while its analog in the variable-energy MPMSA (cf., e.g., [4, Lemma 3]) is substantially more involved.

This constatation has been the principal motivation for the present work. A reader familiar with the techniques of the papers $[4,16,17]$ can see that the multiparticle variant of the DSA (MPDSA) from the present paper is much closer in its logical structure to the FEMSA, yet it leads directly to the VEMSA-type decay bounds on the entire eigenbases in arbitrarily large finite domains.

In preprint [18], which has been a starting point for the present paper, we gave the first proof of exponential decay of multiparticle eigenfunctions in presence of an infinite-range interaction decaying at infinity at a fractional-exponential rate, $r \mapsto \mathrm{e}^{-r^{\zeta}}, 0<\zeta<1$. In a recent work [7], Aizenman and Warzel, further developing the techniques of [6], proved fractional-exponential decay of EF correlators (EFCs) for subexponentially decaying interactions of infinite range. Due to the logical structure of the FMM, the decay analysis of the eigenfunctions is subordinate to that of the EFCs, and this explains why [7] established only a subexponential decay of eigenfunctions. The DSA is free from this limitation, and this allows us to prove a genuine exponential decay of the multiparticle EFs even in the case where the interaction decay is subexponential.

1.3. Novelty. Apart from simplifications of the Multiparticle Multiscale Analysis (MPMSA) developed in [4], the novelty of this paper is that the external random potential is not necessarily IID (with independent and identically distributed values) but is strongly mixing. Earlier publications, including [19], required the common external random potential acting on the particles to be IID.

1.4. Structure of the Paper. The structure of the paper is as follows:

(i) The principal assumptions and main results are stated in Sections 2.4-2.6.

(ii) The deterministic techniques of scaling analysis are presented in Section 3.

(iii) In Section 4, we treat first the case of finite-range interactions, in order to present the logical structure and the methodological advantages of the MPDSA in the most transparent way.

(iv) In Section 7, our analysis is adapted to the interactions of infinite range.

(v) Sections 5 and 6 are devoted to the derivation of spectral and dynamical localization from the final results of the MPDSA.

\section{Preliminaries, Assumptions, and Main Results}

2.1. Configurations of Indistinguishable Particles in $\mathbb{Z}^{1}$. In the first part of this paper, we work with configurations of $N \geq$ 1 quantum particles in the one-dimensional lattice, $\mathscr{Z}=$ $\mathbb{Z}^{1}$. In quantum mechanics, particles of the same kind are considered indistinguishable; more precisely, depending on the nature of the particles, the wave functions describing $N>1$ particles have to be either symmetric (Bose-Einstein quantum statistics) or antisymmetric (Fermi-Dirac quantum statistics). We choose here the fermionic case; this gives rise to slightly simpler notations and constructions.

For clarity, we use often boldface symbols for the objects related to the multiparticle systems.

Quantum states of an $N$-particle fermionic system in $\mathbb{Z}$ are elements of the Hilbert space $\mathscr{H}^{\mathrm{N}}=\mathscr{H}^{N,-}$, formed by all square-summable antisymmetric functions $\Psi: \mathbb{Z}^{N} \rightarrow \mathbb{C}$, with the inner product $\langle\cdot \mid \cdot\rangle$ inherited from the Hilbert space $\ell^{2}\left(\mathbb{Z}^{N}\right)=\left(\mathscr{H}^{1}\right)^{\otimes N}$. In this particular case where the "physical" configuration space is one-dimensional, $\mathscr{H}^{N}$ admits a simple representation which we will use.

First, note that any antisymmetric function

$$
\mathbf{x}=\left(x_{1}, \ldots, x_{N}\right) \longmapsto \mathbf{\Psi}\left(x_{1}, \ldots, x_{N}\right)
$$

vanishes on all hyperplanes $\left\{\mathbf{x}: x_{i}=x_{j}\right\}, 1 \leq i<j \leq N$. Further, any function defined on the "positive sector" $\mathscr{Z}_{>}^{\mathrm{N}}=$ $\left\{\left(x_{1}, \ldots, x_{N}\right): x_{1}>x_{2}>\cdots>x_{N}\right\}$ admits a unique 
antisymmetric continuation to $\mathscr{Z}^{\mathbf{N}}:=\mathbb{Z}^{N}$. An orthonormal basis in $\mathscr{H}^{\mathrm{N}}$ can be chosen in the usual form

$$
\Phi_{\mathbf{a}}(\mathbf{x})=\frac{1}{\sqrt{N !}} \sum_{\pi \in \mathbb{S}_{N}}(-1)^{\pi} \bigotimes_{j=1}^{N} \mathbf{1}_{a_{\pi^{-1}(j)}},
$$

where $\mathbf{a} \in \mathscr{Z}^{\mathbf{N}}$ with $\#\left\{a_{1}, \ldots, a_{N}\right\}=N$ and $\mathbf{1}_{a}$ is the indicator function of the single-point set $\{a\}$. Here $\mathfrak{S}_{N}$ is the symmetric group acting in $\mathbb{Z}^{N}$ by permutations of the particle positions, $\pi(\mathbf{a})=\left(a_{\pi^{-1}(1)}, \ldots, a_{\pi^{-1}(N)}\right)$, and $(-1)^{\pi} \in\{+1,-1\}$ denotes the parity of the permutation $\pi$. It is readily seen that $\mathscr{H}^{\mathbf{N}}$ is unitarily isomorphic to the Hilbert space $\ell^{2}\left(\mathscr{Z}_{>}^{\mathbf{N}}\right)$ of squaresummable functions on $\mathscr{Z}_{>}^{\mathbf{N}}$; equivalently, one can consider square-summable functions on the set $\mathscr{E}_{\geq}^{\mathbf{N}}=\left\{x_{1} \geq \cdots \geq x_{N}\right\}$ vanishing on the boundary $\mathscr{Z}_{=}^{\mathbf{N}}=\left\{\mathbf{x} \in \mathscr{Z}_{\geq}^{\mathbf{N}}: \exists i \neq j, x_{i}=\right.$ $\left.x_{j}\right\}$. Indeed, the isomorphism is induced by the bijection between the orthonormal bases $\left\{\boldsymbol{\Phi}_{\mathbf{a}}\right\}$ and $\left\{\mathbf{1}_{\mathbf{a}}\right\}$ :

$$
\Phi_{\mathrm{a}} \longleftrightarrow \mathbf{1}_{\mathrm{a}}=\mathbf{1}_{a_{1}} \otimes \cdots \otimes \mathbf{1}_{a_{N}}, \quad \mathrm{a} \in \mathscr{Z}_{>}^{\mathrm{N}} .
$$

The subspace $\mathscr{H}^{\mathrm{N}}$ is invariant under any operator commuting with the action of the symmetric group $\mathfrak{\subseteq}_{N}$.

An advantage of the above representation is that $\mathscr{Z}_{>}^{\mathbf{N}}$ inherits its explicit, natural graph structure from $\mathbb{Z}^{N}$. For more general graphs $\mathscr{Z}$, one has to resort to the general construction of the so-called symmetric powers of graphs; see the details in Section 8.

2.2. Fermionic Laplacians. Any unordered finite or countable connected graph $(\mathscr{G}, \mathscr{E})$ with the set of vertices $\mathscr{G}$ and the set of edges $\mathscr{E}$ is endowed with the canonical graph distance $(x, y) \mapsto \mathrm{d}_{\mathscr{G}}(x, y)$ (defined as the length $f$ the shortest path $x \rightsquigarrow y$ over the edges) and with the canonical graph Laplacian $\Delta_{\mathscr{X}}$ :

$$
\begin{aligned}
\left(\Delta_{\mathscr{G}} f\right)(x) & =\sum_{\langle x, y\rangle}(f(y)-f(x)) \\
& =-n_{\mathscr{G}}(x) f(x)+\sum_{\langle x, y\rangle} f(y) ;
\end{aligned}
$$

here $\langle x, y\rangle$ denotes a pair of nearest neighbors, and $n_{\mathscr{G}}(x)$ is the coordination number of the point $x$ in $\mathscr{G}$, that is, the number of its nearest neighbors.

In particular, one can take $\mathscr{G}=\mathbb{Z}^{N}$ (or a subgraph thereof) with the edges $\langle x, y\rangle$ formed by vertices $x, y$ with $|x-y|_{1}:=\left|x_{1}-y_{1}\right|+\cdots+\left|x_{N}-y_{N}\right|=1$. In other words, the vector norm $|\cdot|_{1}$ induces the graph distance on $\mathbb{Z}^{N}$. The Laplacian on $\mathbb{Z}^{N}$, which we will now denote by $\Delta$, can be written as follows:

$$
\Delta=\sum_{j=1}^{N}\left(\bigotimes_{i=1}^{j-1} \mathbf{1}^{(i)}\right) \otimes \Delta^{(j)} \otimes\left(\bigotimes_{k=j+1}^{N} \mathbf{1}^{(k)}\right)
$$

where $\mathbf{1}^{(i)}$ is the identity operator acting on the $i$ th variable and $\Delta^{(j)}$ is the one-dimensional negative lattice Laplacian in the $j$ th variable:

$$
\begin{array}{r}
\left(\Delta^{(j)} f\right)\left(x_{j}\right)=-2 f\left(x_{j}\right)+f\left(x_{j}-1\right)+f\left(x_{j}+1\right), \\
x_{j} \in \mathbb{Z} .
\end{array}
$$

The restriction to the subspace $\mathscr{H}^{\mathrm{N}}$ of antisymmetric functions can be equivalently defined in terms of functions supported by the positive sector $\mathscr{Z}_{>}^{\mathrm{N}}$, hence vanishing on its border $\mathscr{Z}_{=}^{\mathbf{N}}$. Indeed, the matrix elements of $\Delta$ in the basis $\Phi_{\text {a }}$ can be nonzero only for pairs $\Phi_{\mathbf{a}}, \boldsymbol{\Phi}_{\mathbf{b}}$ with $|\mathbf{a}-\mathbf{b}|_{1}=1$, so that, for some $j \in[1, N],\left|a_{j}-b_{j}\right|=1$, while for all $i \neq j, a_{i}=b_{i}$. If $\mathbf{a}, \mathbf{b} \in \mathscr{Z}_{>}^{\mathrm{N}}$, then $\left\langle\boldsymbol{\Phi}_{\mathbf{a}}|\Delta| \boldsymbol{\Phi}_{\mathbf{b}}\right\rangle=(1 / N !)\left\langle\mathbf{1}_{\mathbf{a}}|\Delta| \mathbf{1}_{\mathbf{b}}\right\rangle$. If, say, $\mathbf{a} \in \mathscr{Z}_{=}^{\mathbf{N}}$, then the respective matrix element of the Laplacian's restriction to $\mathscr{Z}_{>}^{\mathrm{N}}$ with Dirichlet boundary conditions on $\mathscr{Z}_{=}^{\mathbf{N}}$ vanishes, and so does the function $\boldsymbol{\Phi}_{\mathrm{a}}$ (which is no longer an element of the basis in $\mathscr{H}^{\mathrm{N}}$ ). Therefore, up to a constant factor, the restriction of the $\mathrm{N}$-particle Laplacian to the fermionic subspace $\mathscr{H}^{\mathrm{N}}$ is unitarily equivalent to the standard graph Laplacian on $\mathscr{Z}_{>}^{\mathrm{N}}$. From this point on, we will work with the latter.

It will be convenient in the course of the scaling analysis to use a different kind of metric on $\mathscr{Z}_{>}^{\mathrm{N}} \subset \mathbb{Z}^{N}$, the max-distance defined by

$$
\boldsymbol{\rho}(\mathbf{x}, \mathbf{y})=\max _{1 \leq j \leq N} \mathrm{~d}_{\mathscr{E}}\left(x_{j}, y_{j}\right),
$$

and to work with the balls relative to the max-distance,

$$
\mathbf{B}_{L}(\mathbf{x})=\{\mathbf{y}: \boldsymbol{\rho}(\mathbf{x}, \mathbf{y}) \leq L\}=\chi_{j=1}^{N} B_{L}\left(x_{j}\right) .
$$

Here $B_{L}(x)=[x-L, x+L] \cap \mathbb{Z}$.

In the positive sector $\mathscr{Z}_{>}^{\mathbf{N}}$, the above factorization of $\boldsymbol{\rho}$ balls is subject to the condition that the RHS of (10) is itself a subset of the positive sector $\mathscr{Z}_{>}^{\mathrm{N}}$ (but the inclusion "c" always holds true). Considering configurations $\mathbf{x}=\left\{x_{1}, \ldots, x_{n}\right\}$ as subsets of $\mathbb{Z}$, one can define the distance between two configurations $\mathbf{x}^{\prime} \in \mathscr{Z}_{>}^{\left(\mathbf{n}^{\prime}\right)}$ and $\mathbf{x}^{\prime \prime} \in \mathscr{Z}_{>}^{\left(\mathbf{n}^{\prime \prime}\right)}$ in a usual way:

$$
\operatorname{dist}\left(\mathbf{x}^{\prime}, \mathbf{x}^{\prime \prime}\right)=\min _{u \in \mathbf{x}^{\prime}} \min _{v \in \mathbf{x}^{\prime \prime}}|u-v| .
$$

Lemma 1. Let $\mathbf{x} \in \mathscr{Z}_{>}^{\mathrm{N}}$ be a union of two subconfigurations $\mathbf{x}^{\prime} \in \mathscr{Z}_{>}^{\left(\mathbf{n}^{\prime}\right)}$ and $\mathbf{x}^{\prime \prime} \in \mathscr{X}_{>}^{\left(\mathbf{n}^{\prime \prime}\right)}$, such that $\operatorname{dist}\left(\mathbf{x}^{\prime}, \mathbf{x}^{\prime \prime}\right)>2 L$. Then the following identity holds true:

$$
\mathbf{B}_{L}^{(N)}(\mathbf{x})=\mathbf{B}_{L}^{\left(n^{\prime}\right)}\left(\mathbf{x}^{\prime}\right) \times \mathbf{B}_{L}^{\left(n^{\prime \prime}\right)}\left(\mathbf{x}^{\prime \prime}\right) .
$$

The proof is straightforward and will be omitted.

The graph distance $\mathrm{d}_{\mathscr{Z}^{\mathrm{N}}}$ will be useful in some geometrical constructions and definitions, referring to the graph structure inherited from $\mathscr{Z}^{\mathrm{N}}$.

Given a subgraph $\Lambda \subset \mathscr{Z}_{>}^{\mathrm{N}}$, we define its internal, external, and the so-called graph (or edge) boundary, in terms 
of the canonical graph distance (below $\Lambda^{c}$ stands for the complement of $\boldsymbol{\Lambda})$ :

$$
\begin{aligned}
\partial^{-} \boldsymbol{\Lambda} & =\left\{\mathbf{y} \in \boldsymbol{\Lambda}: \mathrm{d}_{\mathscr{Z}_{>}^{\mathbb{N}}}\left(\mathbf{y}, \boldsymbol{\Lambda}^{c}\right)=1\right\}, \\
\partial^{+} \boldsymbol{\Lambda} & =\partial^{-} \boldsymbol{\Lambda}^{c} \\
\partial \boldsymbol{\Lambda} & =\left\{(\mathbf{x}, \mathbf{y}) \in \boldsymbol{\Lambda} \times \boldsymbol{\Lambda}^{\mathrm{c}}: \mathrm{d}_{\mathscr{Z}_{>}^{\mathrm{N}}}(\mathbf{x}, \mathbf{y})=1\right\} .
\end{aligned}
$$

We also define the occupation numbers of a site $y$ in the 1-particle configuration space relative to a configuration $\mathbf{x}=\left\{x_{1}, \ldots, x_{N}\right\}$. Namely, define a function $\mathbf{n}_{\mathbf{x}}: \mathbb{Z} \mapsto \mathbb{N}$ by $\mathbf{n}_{\mathbf{x}}(y)=\#\left\{j: x_{j}=y\right\}, y \in \mathscr{X}$.

\subsection{Multiparticle Fermionic Hamiltonians. Given a finite} subset $\Lambda \subset \mathscr{Z}_{>}^{\mathbf{N}}$, let $\Sigma\left(\mathbf{H}_{\Lambda}\right)$ be the spectrum of the operator $\mathbf{H}_{\Lambda}$ and $\mathbf{G}_{\Lambda}(E)=\left(\mathbf{H}_{\Lambda}-E\right)^{-1}$ its resolvent. The matrix elements of resolvents for $E \notin \Sigma\left(\mathbf{H}_{\Lambda}\right)$, in the canonical delta-basis, usually referred to as the Green functions, are denoted by $\mathbf{G}_{\Lambda}(\mathbf{x}, \mathbf{y} ; E)$. In the context of random operators, the dependence upon the element $\omega \in \Omega$ will be often omitted for brevity, unless required or instructive. Similar notations will be used for infinite $\Lambda$.

Given a real-valued function $\mathbf{W}: \mathscr{Z}_{>}^{\mathbf{N}} \rightarrow \mathbb{R}$, we identify it with the operator of multiplication by $\mathbf{W}$ and consider the fermionic $N$-particle random Hamiltonian

$$
\mathbf{H}(\omega)=\mathbf{H}_{0}+\mathbf{W},
$$

where $\mathbf{H}_{0}$ is a second-order finite-difference operator, for example, the negative graph Laplacian $(-\Delta)$. Furthermore, in our model the potential energy is random:

$$
\mathbf{W}(\mathbf{x})=\mathbf{W}(\mathbf{x} ; \omega)=g \mathbf{V}(\mathbf{x} ; \omega)+\mathbf{U}(\mathbf{x}),
$$

where $g \mathbf{V}(\cdot ; \omega)$ is the external random potential energy of the form

$$
g \mathbf{V}(\mathbf{x} ; \omega)=g V\left(x_{1} ; \omega\right)+\cdots+g V\left(x_{N} ; \omega\right),
$$

of amplitude $g>0$, and $V: \mathbb{Z} \times \Omega \rightarrow \mathbb{R}$ is a random field on $\mathbb{Z}$ relative to a probability space $(\Omega, \mathfrak{F}, \mathbb{P})$. The expectation relative to the measure $\mathbb{P}$ will be denoted by $\mathbb{E}[\cdot]$. Our assumptions on $V$ are listed below (cf. (W1)-(W3)). See also Remark 4 where how (W1)-(W3) can be relaxed is explained.

Further, $\mathbf{U}$ is the interaction energy operator; for notational brevity, we assume that it is generated by a two-body interaction potential $U$,

$$
\mathbf{U}(\mathbf{x})=\sum_{i \neq j} U\left(\left|x_{i}-x_{j}\right|\right),
$$

satisfying one of hypotheses (U0)-(U1) (see below).

Note that, without loss of generality, if $\left\|\mathbf{H}_{0}\right\|<\infty$ and $V$ is a.s. bounded, then the random operator has a.s. bounded norm; hence its spectrum is a.s. covered by some bounded interval $I \subset \mathbb{R}$. In Remark 3, we explain that the boundedness of the potential is not crucial for the spectral localization, although the statement of the result on the dynamical localization is to be slightly modified for unbounded potentials.

One can also consider higher-order finite-difference operators $\mathbf{H}_{0}$; this requires only minor technical modifications.
2.4. Assumptions on the Random Potential. We formulate the assumptions in the general situation where the 1-particle configuration space is a graph $\mathscr{Z}$ with graph-distance $\mathrm{d}_{\mathscr{X}}$; this includes the case $\mathscr{Z}=\mathbb{Z}^{1}$.

We assume that the random field $V: \mathscr{Z} \times \Omega \rightarrow \mathbb{R}$ is (possibly) correlated, but strongly mixing; this includes of course the IID potentials treated earlier in $[4,6]$. Let

$$
F_{V, x}(t)=\mathbb{P}\{V(x ; \omega) \leq t\}, \quad x \in \mathscr{Z}
$$

be the marginal probability distribution functions (PDF) of the random field $V$ and

$$
F_{V, x}\left(t \mid \mathfrak{\mho}_{\neq x}\right):=\mathbb{P}\left\{V(x ; \omega) \leq t \mid \mathfrak{\mho}_{\neq x}\right\}
$$

the conditional distribution functions (CDF) of the random field $V$ given the sigma-algebra $\mathfrak{F}_{\neq x}^{V}$ generated by random variables $\{V(y ; \omega), y \neq x\}$. Our assumptions on correlated potentials are as follows:

(W1) The marginal CDFs are uniformly Lipschitz continuous: for some $C \in(0,+\infty)$ and all $s \in(0,1]$

$$
\sup _{x \in \mathscr{E}} \sup _{a \in \mathbb{R}}\left[F_{V, x}\left(a+s \mid \mathfrak{\mho}_{\neq x}^{V}\right)-F_{V, x}\left(a \mid \mathfrak{F}_{\neq x}^{V}\right)\right] \leq C s .
$$

The LHS of (20) is a conditional probability, hence, a random variable, so (20) holds $\mathbb{P}$-a.s.

To formulate the next assumption, introduce the following notation: given a subset $\Lambda \subset \mathscr{Z}$, we denote by $\widetilde{F}_{\Lambda}^{V}$ the sigma-algebra generated by the values of the random potential $\{V(x ; \omega), x \in \Lambda\}$.

(W2) (Rosenblatt strong mixing) For any pair of subsets $B^{\prime}$, $B^{\prime \prime} \subset \mathscr{Z}$ with $\mathrm{d}_{\mathscr{E}}\left(B^{\prime}, B^{\prime \prime}\right) \geq L \geq 1$, any events $\mathscr{E}^{\prime} \in$ $\mathfrak{F}_{B^{\prime}}^{V}, \mathscr{E}^{\prime \prime} \in \mathfrak{F}_{B^{\prime \prime}}^{V}$ and some $C>0$

$$
\left|\mathbb{P}\left\{\mathscr{E}^{\prime} \cap \mathscr{E}^{\prime \prime}\right\}-\mathbb{P}\left\{\mathscr{E}^{\prime}\right\} \mathbb{P}\left\{\mathscr{E}^{\prime \prime}\right\}\right| \leq \mathrm{e}^{-C \ln ^{2} L} .
$$

One can easily check that, for any $p>0, \alpha \in(1,2)$, some $\vartheta \in(0,1), a>0$, and $L_{0} \in \mathbb{N}$ large enough,

$$
\mathrm{e}^{-C \ln ^{2}\left(L_{0}\right)^{\alpha^{k}}}<\left(\left(L_{0}\right)^{\alpha^{k}}\right)^{-a p(1+\theta)^{k}}, \quad k \geq 0 .
$$

The rate of decay of correlations indicated in the RHS of (22) is required to prove dynamical localization bounds with decay rate of EF correlators faster than polynomial, and it can be relaxed to a power-law decay, if one aims to prove only a power-law decay of EF correlators.

Assumptions (W1)-(W2) are sufficient for the proof of spectral and strong dynamical localization in multiparticle systems. In particular, the role of (W1) is to guarantee the eigenvalue concentration (EVC) estimates used in the MPMSA scheme. However, it was discovered in $[4,6]$ that the traditional EVC estimates do not provide all necessary information for efficient decay bounds on the eigenfunctions of multiparticle operators. More precisely, conventional EVC bounds seem so far insufficient for the proof of the exponential decay of eigenfunctions and EF correlators with respect 
to a norm in the configuration space of $N$-particle systems, starting from $N=3$. For this reason, we proposed earlier [20] a new method for comparing spectra of two strongly correlated multiparticle subsystems and improved the EVC estimate from [4]. For the new method to apply, one needs an additional assumption on the random potential field which we will describe now.

First, introduce the following notations. Given a finite subset $Q \subset \mathscr{Z}$, we denote by $\xi_{Q}(\omega)$ the sample mean of the random field $V$ over $Q$,

$$
\xi_{Q}(\omega):=|Q|^{-1} \sum_{x \in Q} V(x, \omega)
$$

and define the "fluctuations" of $V$ relative to the sample mean, $\eta_{x}:=V(x, \omega)-\xi_{\mathrm{Q}}(\omega), x \in Q$. Denote by $\mathfrak{F}_{\mathrm{Q}}$ the sigma-algebra generated by $\left\{\eta_{x}, V_{y}: x \in Q, y \notin Q\right\}$.

We will assume that the random field $V$ fulfills the following condition:

(W3) There exist $C^{\prime}, A^{\prime}, b^{\prime} \in(0,+\infty)$ such that for any finite subset $Q \subset \mathscr{Z}$ of cardinality $|Q|$ and any $\mathfrak{F}_{Q^{-}}$-measurable function $\lambda$ and all $s \in(0,1]$

$$
\mathbb{P}\left\{\xi_{\mathrm{Q}}(\omega) \in[\lambda(\omega), \lambda(\omega)+s]\right\} \leq C^{\prime}|Q|^{A^{\prime}} s^{b^{\prime}} .
$$

In the particular case of a Gaussian IID field, for example, with zero mean and unit variance, it is well-known from the standard courses of probability that $\xi_{\mathrm{Q}}$ is independent of the "fluctuations" $\eta_{x}$, so it is Gaussian with variance $|Q|^{-1}$ (if $V(x ; \omega)$ has unit variance) that its probability density $p_{Q}$ is bounded, although $\left\|p_{Q}\right\|_{\infty} \sim|Q|^{1 / 2} \rightarrow \infty$ as $|Q| \rightarrow \infty$. Property (W3) has been recently proven for a larger class of IID potentials (cf. [21]).

Specifically, (W3) has been established in [21] for IID random potentials with marginal probability measure $\mu$ which admits a representation of the form $\mu=\mu_{1} * \mu_{2}$, where $\mu_{2}$ is an arbitrary probability measure on $\mathbb{R}$ and $\mu_{1}$ is supported by some bounded interval $[a, b]$ and admits probability density $\rho_{1}(\cdot)$ which fulfills the following condition:

$$
\begin{aligned}
0 & <\rho_{*} \leq \rho_{1}(t) \leq \bar{\rho}<\infty, \\
\frac{\rho_{1}^{\prime}(t)}{\rho_{1}(t)} & \leq C_{1}<\infty,
\end{aligned}
$$

$\forall t \in(a, b)$.

A prototypical example is the uniform distribution on $[a, b]$.

2.5. Assumptions on the Interaction Potential. We assume that the interaction potential $U$ generating the interaction energy $\mathrm{U}$ satisfies one of the following decay conditions:

(U0) There exists $r_{0}<\infty$ such that

$$
U(r)=0, \quad \forall r \geq r_{0} .
$$

(U1) There are some $C \in(0,+\infty), \delta \in(0,1 / 14)$, and $\zeta \in$ $(0, \delta /(1+\delta))$ such that

$$
|U(r)| \leq C \mathrm{e}^{-c r^{1-\zeta}}, \quad \forall r \geq 0 .
$$

Naturally, (U0) $\Rightarrow$ (U1). We will prove the multiparticle localization first under the strongest assumption (U0) (leading to a simpler proof), to illustrate the general structure of the DSA procedure, and then extend the proof to the infiniterange interactions satisfying (U1).

\subsection{Main Results}

Theorem 2. Assume that a bounded random field $V$ fulfills conditions (W1)-(W3), and the interaction $\mathrm{U}$ fulfills one of the conditions (U0) and (U1). There exists $g_{0} \in(0,+\infty)$ such that for $|g| \geq g_{0}$ the following holds true.

(A) With probability one, the fermionic operator $\mathbf{H}(\omega)=$ $-\Delta+g \mathbf{V}(\omega)+\mathbf{U}$ has pure point spectrum and all its eigenfunctions $\Psi_{j}(\omega)$ are exponentially decaying at infinity: for each $\Psi_{j}$, some $\widehat{\mathbf{x}}_{j}$, and all $\mathbf{x}$ with $\rho\left(\widehat{\mathbf{x}}_{j}, \mathbf{x}\right)$ large enough,

$$
\left|\Psi_{j}(\mathbf{x}, \omega)\right| \leq \mathrm{e}^{-m \boldsymbol{\rho}\left(\widehat{\mathbf{x}}_{j}, \mathbf{x}\right)}, \quad m>0 .
$$

(B) For some $C, a, c>0$ and any $\mathbf{x}, \mathbf{y} \in \mathscr{Z}_{>}^{\mathbf{N}}$

$$
\mathbb{E}\left[\sup _{t \in \mathbb{R}}\left|\left\langle\mathbf{1}_{\mathbf{y}}\left|\mathrm{e}^{-\mathrm{it} \mathbf{H}(\omega)}\right| \mathbf{1}_{\mathbf{x}}\right\rangle\right|\right] \leq C \mathrm{e}^{-a \ln ^{1+c} \boldsymbol{\rho}(\mathbf{x}, \mathbf{y})} .
$$

Consequently, for any finite subset $\mathbf{K} \subset \mathscr{Z}_{>}^{\mathbf{N}}$

$$
\mathbb{E}\left[\sup _{t \in \mathbb{R}}\left\|\mathrm{e}^{(a / 2) \ln ^{1+c} \mathbf{X}} \mathrm{e}^{-\mathrm{i} t \mathbf{H}(\omega)} \mathbf{1}_{\mathbf{K}}\right\|\right]<\infty,
$$

where $\mathbf{X}_{\mathbf{K}}$ is the operator of multiplication by the function $\mathbf{x} \mapsto(\rho(\mathbf{K}, \mathbf{x})+1)$.

Remark 3. The assumption of boundedness of the random potential is inessential for the proof of spectral localization (exponential decay of all eigenfunctions) and for the analog of the decay bound (29) on eigenfunction correlators in arbitrary large but finite subset $\Lambda \subset \mathscr{Z}_{>}^{\mathrm{N}}$ of the $N$-particle configuration space (cf. Corollary 27). Without any condition on the rate of decay of the tail probabilities $\mathbb{P}\{|V(x ; \omega)| \geq s\}$ as $s \rightarrow+\infty$, the propagator $\mathrm{e}^{-\mathrm{i} t \mathbf{H}(\omega)}$ in (29)-(30) has to be replaced with $P_{I}(\mathbf{H}(\omega)) \mathrm{e}^{-\mathrm{i} t \mathbf{H}(\omega)}$, where $I \subset \mathbb{R}$ is an arbitrary bounded interval, and $P_{I}(\mathbf{H}(\omega))$ is the spectral projection for $\mathbf{H}(\omega)$ on $I$.

Remark 4. Exponential spectral localization can be proved without condition (W3), with (W1) relaxed to Hölder continuity. Indeed, one can use the EVC bound from [4, Lemma 2], valid for the pairs of balls distant in the so-called Hausdorff pseudo-metric $\mathrm{d}_{\mathscr{H}}$ in the multiparticle configuration space, 
defined as follows: for the configurations $\mathbf{x}=\left\{x_{1}, \ldots, x_{N}\right\}$, $\mathbf{y}=\left\{y_{1}, \ldots, y_{N}\right\}$,

$$
\mathrm{d}_{\mathscr{H}}(\mathbf{x}, \mathbf{y}):=\max \left[\max _{i} \mathrm{~d}_{\mathscr{E}}\left(x_{i}, \mathbf{y}\right), \max _{i} \mathrm{~d}_{\mathscr{Z}}\left(y_{i}, \mathbf{x}\right)\right],
$$

where

$$
\mathrm{d}_{\mathscr{Z}}(x, \mathbf{y})=\min _{j} \mathrm{~d}_{\mathscr{Z}}\left(x, y_{j}\right)
$$

In this case, the decay bounds on the eigenfunction correlators would also be established with respect to $\mathrm{d}_{\mathscr{H}}$ replacing the max-distance $\rho$ :

$$
\mathbb{E}\left[\sup _{t \in \mathbb{R}}\left|\left\langle\mathbf{1}_{\mathbf{y}}\left|\mathrm{e}^{-\mathrm{i} t \mathbf{H}(\omega)}\right| \mathbf{1}_{\mathbf{x}}\right\rangle\right|\right] \leq C(\mathbf{x}) \mathrm{e}^{-a \ln ^{1+c} \mathrm{~d}_{\mathscr{H}}(\mathbf{x}, \mathbf{y})}
$$

Observe that the RHS bound is not uniform as in (29) but depends upon $\mathbf{x}$; this is an artefact of the EVC bounds based on the Hausdorff distance (cf. $[4,6,7,17])$. Of course, the roles of $\mathbf{x}$ and $\mathbf{y}$ in the LHS of (33) are similar, so the factor $C(\mathbf{x})$ in the RHS can be replaced by $C(\mathbf{y})$ or, more precisely, by some function $C^{\prime}(\operatorname{diam}(\mathbf{x}) \wedge \operatorname{diam}(\mathbf{y}))$. As to the proofs, they require only minor notational modifications, for they adapt easily to any kind of pseudometric in the $N$-particle configuration space for which an EVC bound of the general form (64) can be proved, with some $h_{L}(s) \leq C L^{A} s^{n}, C, A \in$ $(0,+\infty)$, and $b \in(0,1]$.

\section{Deterministic Bounds}

3.1. Geometric Resolvent Inequality. The most essential part of the Direct Scaling Analysis concerns the finite-volume approximations $\mathbf{H}_{\Lambda}(\omega)$ of the random Hamiltonian $\mathbf{H}(\omega)$, acting in finite-dimensional spaces $\mathscr{H}_{\Lambda}$, with $\Lambda \subset \mathscr{Z}_{>}^{\mathrm{N}},|\Lambda| \equiv$ card $\boldsymbol{\Lambda}<\infty$. We define

$$
\mathbf{H}_{\Lambda}(\omega):=\mathbf{1}_{\Lambda} \mathbf{H}(\omega) \mathbf{1}_{\Lambda} \uparrow \mathscr{H}_{\Lambda},
$$

where the indicator function $\mathbf{1}_{\Lambda}$ is identified with the operator of multiplication by $\mathbf{1}_{\Lambda} \cdot \mathbf{H}_{\Lambda}$ is a bounded, finite-dimensional Hermitian operator, so its spectrum $\Sigma\left(\mathbf{H}_{\Lambda}\right)$ is real.

Operator $(-\Delta)$ can be represented as follows:

$$
\begin{aligned}
-\Delta & =-n_{\mathscr{Z}^{\mathbf{N}}}+\sum_{\langle\mathbf{x}, \mathbf{y}\rangle}\left(\Gamma_{\mathbf{x y}}+\Gamma_{\mathbf{y x}}\right), \\
\left(\Gamma_{\mathbf{x y}} f\right)(\mathbf{x}) & :=\delta_{\mathbf{x y}} f(\mathbf{y}),
\end{aligned}
$$

and $\delta_{\mathbf{x y}}$ is the Kronecker symbol.

Recall that we denote by $\mathbf{G}_{\Lambda}(E)=\left(\mathbf{H}_{\Lambda}-E\right)^{-1}$ the resolvent of $\mathbf{H}_{\Lambda}$ and by $\mathbf{G}(\mathbf{x}, \mathbf{y} ; E)$ the matrix elements thereof in the standard delta-basis (the Green functions).

The so-called Geometric Resolvent Inequality (GRI) for the Green functions can be easily deduced from the second resolvent identity (we omit $E$ for brevity):

$$
\begin{aligned}
& \left|\mathbf{G}_{\mathbf{B}_{L}}(\mathbf{x}, \mathbf{y})\right| \\
& \quad \leq C_{\ell} \max _{\mathbf{v} \in \partial^{-} \mathbf{B}_{\ell}(\mathbf{x})}\left|\mathbf{G}_{\mathbf{B}_{\ell}(\mathbf{x})}(\mathbf{x}, \mathbf{v})\right| \max _{\mathbf{v}^{\prime} \in \partial^{+} \mathbf{B}_{\ell}(\mathbf{x})}\left|\mathbf{G}_{\mathbf{B}_{L}}\left(\mathbf{v}^{\prime}, \mathbf{y}\right)\right|,
\end{aligned}
$$

where

$$
C_{\ell}=\left|\partial \mathbf{B}_{\ell}(\mathbf{v})\right| \leq C(N) \ell^{N}
$$

See, for example, [22]. Clearly, (36) implies the inequality

$$
\begin{aligned}
& \left|\mathbf{G}_{B_{L}}(\mathbf{x}, \mathbf{y})\right| \\
& \quad \leq\left(C_{\ell} \max _{\rho(\mathbf{x}, \mathbf{v})=\ell}\left|\mathbf{G}_{\mathbf{B}_{\ell}(\mathbf{x})}(\mathbf{x}, \mathbf{v})\right|\right) \max _{\rho\left(\mathbf{u}, \mathbf{v}^{\prime}\right) \leq \ell+1}\left|\mathbf{G}_{\mathbf{B}_{L}}\left(\mathbf{v}^{\prime}, \mathbf{y}\right)\right| .
\end{aligned}
$$

Sometimes we use another inequality stemming from (36):

$$
\begin{aligned}
& \left|\mathbf{G}_{\mathbf{B}_{L}}(\mathbf{x}, \mathbf{y} ; E)\right| \\
& \quad \leq C_{\ell}\left\|\mathbf{G}_{\mathbf{B}_{\ell}(\mathbf{u})}(E)\right\| \max _{\mathbf{v}: \rho(\mathbf{u}, \mathbf{v}) \leq \ell+1}\left|\mathbf{G}_{\mathbf{B}_{L}}(\mathbf{v}, \mathbf{y} ; E)\right| .
\end{aligned}
$$

Similarly, for the solutions $\psi$ of the eigenfunction equation $\mathbf{H} \psi=E \boldsymbol{\psi}$ one has, with $\mathbf{x} \in \mathbf{B}_{\ell}(\mathbf{u})$,

$$
|\psi(\mathbf{x})| \leq C_{\ell}\left\|\mathbf{G}_{\mathbf{B}_{\ell}(\mathbf{x})}(E)\right\| \max _{\mathbf{y}: \rho(\mathbf{x}, \mathbf{y}) \leq \ell+1}|\psi(\mathbf{y})|,
$$

provided that $E$ is not an eigenvalue of the operator $\mathbf{H}_{\mathbf{B}_{\ell}(\mathbf{u})}$.

3.2. Dominated Decay Bounds for the Green Functions and Eigenfunctions. The analytic statements of this subsection apply indifferently to any discrete Schrödinger operators, on fairly general graphs, regardless of their single- or multiparticle structure of the potential energy. To avoid any confusion, in this subsection we denote the underlying graph by $\mathscr{G}$; in applications to $N$-particle models in $\mathscr{Z}$, one has to set $\mathscr{G}=$ $\mathscr{Z}_{>}^{\mathbf{N}}$.

Definition 5. Fix an integer $\ell \geq 0$ and a number $q \in(0,1)$. Consider a finite connected subgraph $\Lambda \subset \mathscr{G}$ and a ball $B_{R}(u) \subsetneq \Lambda$. A function $f: B_{R}(u) \rightarrow \mathbb{R}_{+}$is called $(\ell, q)$ dominated in $B_{R}(u)$ if for any ball $B_{\ell}(x) \subset \Lambda_{R}(u)$ one has

$$
f(x) \leq q \max _{y: \mathrm{d}(x, y) \leq \ell} f(y) .
$$

Lemma 6. Let be given integers $L \geq \ell \geq 0$ and a number $q \in(0,1)$. If $f: \Lambda \rightarrow \mathbb{R}_{+}$on a finite connected graph $\Lambda$ is $(\ell, q)$-dominated in a ball $B_{L}(x) \subsetneq \Lambda$, then

$$
\begin{aligned}
f(x) & \leq q^{\lfloor(L+1) /(\ell+1)\rfloor} \mathscr{M}(f, B) \\
& \leq q^{(L-\ell) /(\ell+1)} \mathscr{M}(f, B), \\
\mathscr{M}(f, \Lambda) & :=\max _{x \in \Lambda}|f(x)| .
\end{aligned}
$$

Proof. The claim follows from (41) by induction ("radial descent"):

$$
\begin{aligned}
f(x) & \leq q \mathscr{M}\left(f, B_{\ell+1}\right) \leq \cdots \leq q^{j} \mathscr{M}\left(f, B_{j(\ell+1)}\right) \leq \cdots \\
& \leq q^{\lfloor(L+1) /(\ell+1)\rfloor} \mathscr{M}(f, B) .
\end{aligned}
$$


Lemma 7. Let $\Lambda$ be a finite connected graph and $f: \Lambda \times \Lambda \rightarrow$ $\mathbb{R}_{+},\left(x^{\prime}, x^{\prime \prime}\right) \mapsto f\left(x^{\prime}, x^{\prime \prime}\right)$, a function which is separately $(\ell, q)$ dominated in $x^{\prime} \in B_{r^{\prime}}\left(u^{\prime}\right) \subsetneq \Lambda$ and in $x^{\prime \prime} \in B_{r^{\prime \prime}}\left(u^{\prime \prime}\right) \subsetneq \Lambda$, with $\mathrm{d}\left(u^{\prime}, u^{\prime \prime}\right) \geq r^{\prime}+r^{\prime \prime}+2$. Then

$$
\begin{aligned}
f\left(u^{\prime}, u^{\prime \prime}\right) & \leq q^{\left\lfloor\left(r^{\prime}+1\right) /(\ell+1)\right\rfloor+\left\lfloor\left(r^{\prime \prime}+1\right) /(\ell+1)\right\rfloor} \mathscr{M}(f, B) \\
& \leq q^{\left(r^{\prime}+r^{\prime \prime}-2 \ell\right) /(\ell+1)} \mathscr{M}(f, B) .
\end{aligned}
$$

Proof. Fix any point $y^{\prime \prime} \in B_{r^{\prime \prime}+1}\left(u^{\prime \prime}\right)$ and consider the function

$$
f_{y^{\prime \prime}}: y^{\prime} \longmapsto f\left(y^{\prime}, y^{\prime \prime}\right)
$$

which is $(\ell, q)$-dominated in $B_{r^{\prime}}\left(u^{\prime}\right) \subsetneq \Lambda$, so by Lemma 6 ,

$$
f_{y^{\prime \prime}}\left(u^{\prime}\right)=f\left(u^{\prime}, y^{\prime \prime}\right) \leq q^{\left\lfloor\left(r^{\prime}+1\right) /(\ell+1)\right\rfloor} \mathscr{M}(f, \Lambda \times \Lambda) .
$$

Now introduce the function $\widetilde{f}_{u^{\prime}}: y^{\prime \prime} \mapsto f\left(u^{\prime}, y^{\prime \prime}\right)$ which is $(\ell, q)$-dominated in $B_{r^{\prime \prime}}\left(u^{\prime \prime}\right) \subsetneq \Lambda$ and bounded by the RHS of (46). Applying again Lemma 6, the claim follows.

The relevance of the above notions and results is illustrated by Lemma 10, stated in the next subsection. It could be stated in a general form, for arbitrary countable graphs, but its formulation relies on the notions of "nonsingular" and "nonresonant" balls. For our model, these are given in Definitions 8 and 9. However, the proof given in [14] (cf. [14, Lemma 4]) applies indifferently to arbitrary graphs.

3.3. Localization and Tunneling in Finite Balls. From this point on, we will work with a sequence of length scales, positive integers $\left\{L_{k}, k \geq 0\right\}$ defined recursively by $L_{k+1}=$ $\left\lceil L_{k}^{\alpha}\right\rceil, L_{0}>2$. For clarity, we keep the value $\alpha=4 / 3$; observe that $\alpha^{2}<2$. In several arguments we will require the initial scale $L_{0}$ to be large enough.

Definition 8. Given a sample of the random potential $V(\cdot ; \omega)$, a ball $\mathbf{B}_{L}(\mathbf{u})$ is called

(i) $E$-nonresonant $(E-\mathrm{NR})$ if $\left\|\mathbf{G}_{\mathbf{B}}(E ; \omega)\right\| \leq \mathrm{e}^{+L^{\beta}}$ and $E$ resonant $(E-\mathrm{R})$ otherwise;

(ii) completely $E$-nonresonant $(E$-CNR) if it does not contain any $E$-R ball $\mathbf{B}_{\ell}(\mathbf{u}) \subseteq \mathbf{B}_{L}(\mathbf{u})$ with $\ell \geq L^{1 / \alpha}$ and $E$-partially resonant (E-PR) otherwise.

Definition 9. Given a sample $V(\cdot ; \omega)$, a ball $\mathbf{B}_{L}(\mathbf{u})$ is called $(E, m)$-nonsingular $((E, m)$-NS $)$ if

$$
\max _{\mathbf{y} \in \partial^{-} \mathbf{B}_{L}(\mathbf{u})}\left|\mathbf{G}_{\mathbf{B}_{L}(\mathbf{u})}(\mathbf{u}, \mathbf{y} ; E ; \omega)\right| \leq \mathrm{e}^{-\gamma(m, L) L+2 L^{\beta}},
$$

where

$$
\gamma(m, L):=m\left(1+L^{-\tau}\right), \quad \tau=\frac{1}{8} .
$$

Otherwise, it is called $(E, m)$-singular $((E, m)-S)$.
Lemma 10 (cf. [14, Lemma 4]). Consider a ball $\mathbf{B}_{L_{k}}(\mathbf{u})$ and an operator $\mathbf{H}=\mathbf{H}_{\mathbf{B}_{L_{k}}(\mathbf{u})}$ with fixed (nonrandom) potential $\mathbf{W}$. Let $\left\{\boldsymbol{\psi}_{j}, j=1, \ldots,\left|\mathbf{B}_{L_{k}}(\mathbf{u})\right|\right\}$ be the normalized eigenfunctions of H. Pick a pair of points $\mathbf{x}_{0}, \mathbf{y}_{0} \in \mathbf{B}_{L_{k}}(\mathbf{u})$ with $\mathrm{d}\left(\mathbf{x}_{0}, \mathbf{y}_{0}\right)>L_{k-1}+1$ and an integer

$$
R \in\left[L_{k-1}, \mathrm{~d}\left(\mathbf{x}_{0}, \mathbf{y}_{0}\right)-L_{k-1}+1\right] .
$$

Suppose that any ball $\mathbf{B}_{L_{k-1}}(\mathbf{v}) \subset \mathbf{B}_{R}\left(\mathbf{x}_{0}\right)$ is $(E, m)-N S$, and set

$$
q=\mathrm{e}^{-\gamma(m, \ell) \ell}
$$

Then

(A) the kernel $\Pi_{\psi_{j}}\left(\mathbf{x}, \mathbf{y}_{0}\right)$ of the spectral projection $\Pi_{\psi_{j}}=$ $\left|\psi_{j}\right\rangle\left\langle\psi_{j}\right|$, considered as a function of $\mathbf{x}$, is $(\ell+1, q)$ dominated in $\mathbf{x} \in \mathbf{B}_{R}\left(\mathbf{x}_{0}\right)$ and globally bounded by 1 ;

(B) if the ball $\mathbf{B}_{L_{k}}(\mathbf{u})$ is also E-NR, then the Green function

$$
\mathbf{B}_{R}\left(\mathbf{x}_{0}\right) \ni \mathbf{x} \longmapsto\left|\mathbf{G}_{\mathbf{B}_{L_{k}}(\mathbf{u})}(\mathbf{x}, \mathbf{y} ; E)\right|
$$

is $(\ell+1, q)$-dominated in $\mathbf{x} \in \mathbf{B}_{R}\left(\mathbf{x}_{0}\right)$ and globally bounded by $\mathrm{e}^{L^{\beta}}$.

By Hermitian symmetry of the Green functions, the counterparts of assertions (A) and (B) relative to the second argument, $\mathbf{y} \in \mathbf{B}_{R}\left(\mathbf{y}_{0}\right)$, also hold true, with $\mathbf{x}=\mathbf{x}_{0}$ fixed.

In the next definition, we use a parameter $\varrho=(\alpha-1) / 2$; with $\alpha=4 / 3$, one obtains $\varrho=1 / 6$ and $(1+\varrho) / \alpha=7 / 8$.

Definition 11. A ball $\mathbf{B}_{L}(\mathbf{u})$ is called $m$-localized $(m-\mathscr{L} o c$, in short) if every eigenfunction $\psi_{j}$ of the operator $\mathbf{H}_{\mathbf{B}_{L}(\mathbf{u})}$ satisfies

$$
\left|\psi_{j}(\mathbf{x}) \psi_{j}(\mathbf{y})\right| \leq \mathrm{e}^{-\gamma(m, L) \rho(\mathbf{x}, \mathbf{y})}
$$

for any points $\mathbf{x}, \mathbf{y} \in \mathbf{B}_{L}(\mathbf{u})$ with $\rho(\mathbf{x}, \mathbf{y}) \geq L^{(1+\varrho) / \alpha} \equiv L^{7 / 8}$.

Definition 12. A pair of balls $\mathbf{B}_{L}(\mathbf{x}), \mathbf{B}_{L}(\mathbf{y})$ is called distant iff $\rho(\mathbf{x}, \mathbf{y}) \geq C_{N} L$, with $C_{N}=2 A_{N}+3, A_{N}=4 N$.

(The role of the constants $A_{N}$ and $C_{N}$ will become clear in Section 4.3.)

Definition 13. A ball $\mathbf{B}_{\ell^{\alpha}}(\mathbf{u})$ is called $m$-tunneling $(m-\mathrm{T})$ if it contains a pair of distant $m-\mathcal{N} \mathscr{L}$ oc balls $\mathbf{B}_{\ell}(\mathbf{x}), \mathbf{B}_{\ell}(\mathbf{y})$ and $m$ nontunneling ( $m$-NT), otherwise.

It is to be emphasized that, unlike the property of $E$ resonance or $(E, m)$-singularity, the tunneling property is not related to a specific value of energy $E$, and tunneling even in $a$ single ball occurs with a small probability. This is an advantage of the DSA compared to the fixed-energy MSA; yet, as was said in Introduction, the DSA provides final decay bounds for the Green functions in an entire range of energies, like the variable-energy MSA. However, unlike the MSA, the DSA also provides bounds on the eigenbases in the finite balls.

Lemma 14. Let be given $m$-localized ball $\mathbf{B}=\mathbf{B}_{L}(\mathbf{u})$. If, for some $E \in \mathbb{R}, \mathbf{B}$ is also $E-N R$, then it is $(E, m)-N S$. 
Proof. The matrix elements of the resolvent $\mathbf{G}_{\mathbf{B}}(E)$ can be assessed as follows:

$$
\left|\mathbf{G}_{\mathbf{B}}(\mathbf{x}, \mathbf{y} ; E)\right| \leq \sum_{E_{j} \in \Sigma\left(\mathbf{H}_{\mathbf{B}}\right)} \frac{\left|\psi_{j}(\mathbf{x})\right|\left|\psi_{j}(\mathbf{y})\right|}{\left|E-E_{j}\right|} .
$$

Here $\Sigma\left(\mathbf{H}_{\mathbf{B}}\right)$ is the spectrum of the operator $\mathbf{H}_{\mathbf{B}}$.

If $\operatorname{dist}\left(E, \Sigma\left(\mathbf{H}_{\mathbf{B}}\right)\right) \geq \mathrm{e}^{-L^{\beta}}$ and $\ln (2 L+1)^{N} \leq L^{\beta}$, then the $m$ - $\mathscr{L}$ oc property implies

$$
\left|\mathbf{G}_{\mathbf{B}}(\mathbf{x}, \mathbf{y} ; E)\right| \leq \mathrm{e}^{-\gamma(m, L) L+L^{\beta}+\ln |\mathbf{B}|} \leq \mathrm{e}^{-\gamma(m, L) L+2 L^{\beta}} .
$$

Observe that, with $m \geq 1$ and $1-\tau>\beta$, one has for $L_{0}$ large enough (hence, $L_{k}$ large enough, $k \geq 0$ )

$$
\begin{aligned}
m\left(1+L_{k}^{-\tau}\right) L_{k}-2 L_{k}^{\beta} & =m L_{k}+m L_{k}^{1-\tau}-2 L_{k}^{\beta} \\
& \geq m\left(1+\frac{1}{2} L_{k}^{-\tau}\right) L_{k} .
\end{aligned}
$$

We will see that the condition $m \geq 1$ is fulfilled for $|g|$ large enough (cf. Lemma 17).

Notice that the above proof uses the following fact: the cardinality of an $N$-particle ball of radius $L$ is polynomially bounded in $\mathrm{L}$. This will be used in Section 8. The same remains true for the symmetric powers of graphs $\mathscr{Z}$ satisfying the growth condition (129); this includes all lattices $\mathscr{Z}=\mathbb{Z}^{d}$, $d \geq 1$.

Lemma 15. There is $\widetilde{L}^{(1)} \in \mathbb{N}$ such that for $L_{0} \geq \widetilde{L}^{(1)}$

(A) if a ball $\mathbf{B}_{L_{k+1}}(\mathbf{u})$ is E-CNR and contains no pair of distant $(E, m)-S$ balls $\mathbf{B}_{L_{k}}(\mathbf{v}), \mathbf{B}_{L_{k}}(\mathbf{w})$, then it is also $(E, m)-N S$;

(B) if a ball $\mathbf{B}_{L_{k+1}}(\mathbf{u})$ is $m-N T$ and $E-C N R$, then it is also $(E, m)-N S$.

Proof. (A) By assumption, either $\mathbf{B}_{L_{k+1}}(\mathbf{u})$ is $E$-CNR and contains no $(E, m)-S$ ball of radius $L_{k}$ or there is a point $\mathbf{w} \in \mathbf{B}_{L_{k+1}}(\mathbf{u})$ such that any ball $\mathbf{B}_{L_{k}}(\mathbf{v}) \subset \mathbf{B}_{L_{k}}(\mathbf{u})$ with $\rho(\mathbf{w}, \mathbf{v}) \geq C_{N} L_{k}$ is $(E, m)$-NS. We will obtain the required bound without using the balls with $\rho(\mathbf{w}, \mathbf{v})<C_{N} L_{k}$.

In the former case, such an exclusion is unnecessary, but in order to treat both situations with one argument, we can formally set $\mathbf{w}=\mathbf{u}$ (in fact, the choice of the center of a "fictitious" singular ball does not really matter).

Fix arbitrary points $\mathbf{x}, \mathbf{y}$ with $R:=\rho(\mathbf{x}, \mathbf{y})>L_{k}^{1+\varrho}$; as before, $\varrho=1 / 6$. By the triangle inequality,

$$
\begin{aligned}
& \rho\left(\mathbf{x}, \mathbf{B}_{\left(C_{N}-1\right) L_{k}}(\mathbf{w})\right)+\rho\left(\mathbf{y}, \mathbf{B}_{\left(C_{N}-1\right) L_{k}}(\mathbf{w})\right) \\
& \quad \geq R-\left(2 C_{N}-2\right) L_{k} .
\end{aligned}
$$

Assume first that

$$
\begin{aligned}
r^{\prime} & :=\rho\left(\mathbf{x}, \mathbf{B}_{\left(C_{N}-1\right) L_{k}}(\mathbf{w})\right) \geq L_{k}+1, \\
r^{\prime \prime} & :=\rho\left(\mathbf{y}, \mathbf{B}_{\left(C_{N}-1\right) L_{k}}(\mathbf{w})\right) \geq L_{k}+1 .
\end{aligned}
$$

Inside $\mathbf{B}_{r^{\prime}}(\mathbf{x}) \cup \mathbf{B}_{r^{\prime \prime}}(\mathbf{y})$, all the balls of radius $L_{k}$ are automatically disjoint from $\mathbf{B}_{2 L_{k}}(\mathbf{w})$; thus they are $(E, m)$-NS. Furthermore,

$$
r^{\prime}+r^{\prime \prime} \geq R-2\left(C_{N}-1\right) L_{k}-2 \geq R-2 C_{N} L_{k} .
$$

Consider the set $\mathbf{B}=\mathbf{B}_{r^{\prime}}(\mathbf{x}) \times \mathbf{B}_{r^{\prime \prime}}(\mathbf{y})$ and the function $f: \mathbf{B} \rightarrow \mathbb{C}$ defined by $f\left(\mathbf{x}^{\prime}, \mathbf{x}^{\prime \prime}\right)=\mathbf{G}_{\mathbf{B}_{L_{k+1}}}\left(\mathbf{x}^{\prime}, \mathbf{x}^{\prime \prime} ; E\right)$. Since $E$ is not a pole of the resolvent $\mathbf{G}_{\mathbf{B}_{L_{k+1}}(\mathbf{u})}(\cdot)$, its matrix elements are well defined, hence bounded on a finite set. By Lemma $10, f$ is $\left(L_{k}, q\right)$-dominated both in $\mathbf{x}^{\prime}$ and in $\mathbf{x}^{\prime \prime}$, with $q \leq \mathrm{e}^{-\gamma\left(m, L_{k}, n\right)}$. Therefore, one can write, with the convention $-\ln 0=+\infty$, using Lemma 7,

$$
\begin{aligned}
& -\ln f(\mathbf{u}, \mathbf{y}) \\
& \geq-\ln \left[\left(\mathrm{e}^{-m\left(1+(1 / 2) L_{k}^{-\tau}\right) L_{k}}\right)^{\left(R-2 C_{N} L_{k}-2 L_{k}\right) /\left(L_{k}+1\right)} \mathrm{e}^{L_{k+1}^{\beta}}\right] \\
& =m\left(1+\frac{1}{2} L_{k}^{-\tau}\right) \frac{L_{k}}{L_{k}+1} R\left(1-3 \mathrm{C}_{N} R^{-1} L_{k}\right)-L_{k+1}^{\beta} \\
& =m R\left[\left(1+\frac{1}{2} L_{k}^{-\tau}\right)\left(1-L_{k}^{-1}\right)\left(1-3 C_{N} L_{k}^{-\varrho}\right)\right. \\
& \left.-\frac{L_{k+1}^{\beta}}{m R}\right] \geq m\left(1+\frac{1}{4} L_{k}^{-\tau}\right) R \geq \gamma\left(m, L_{k+1}\right) R .
\end{aligned}
$$

If $r^{\prime}=0$ (resp., $r^{\prime \prime}=0$ ), the required bound follows from the dominated decay of the function $f\left(\mathbf{x}^{\prime}, \mathbf{x}^{\prime \prime}\right)$ in $\mathbf{x}^{\prime \prime}$ (resp., in $\mathbf{x}^{\prime}$ ).

(B) Assume otherwise. Then, by assertion (A), the E-CNR ball $\mathbf{B}_{L_{k+1}}(\mathbf{u})$ must contain a pair of distant $(E, m)-S$ balls $\mathbf{B}_{L_{k}}(\mathbf{x}), \mathbf{B}_{L_{k}}(\mathbf{y})$. Both of them are $E-N R$, since $\mathbf{B}_{L_{k+1}}(\mathbf{u})$ is $E$ CNR. By virtue of Lemma 14 , both $\mathbf{B}_{L_{k}}(\mathbf{x})$ and $\mathbf{B}_{L_{k}}(\mathbf{y})$ must be $m-\mathscr{N} \mathscr{L}$ oc, so that $\mathbf{B}_{L_{k+1}}(\mathbf{u})$ must be $m$-T, which contradicts the hypothesis.

Lemma 16. There is $\widetilde{L}^{(2)} \in \mathbb{N}$ such that, for all $L_{0} \geq \widetilde{L}^{(2)}$, if for any $E \in \mathbb{R}$ a ball $\mathbf{B}_{L_{k+1}}$ (u) contains no pair of distant $(E, m)-S$ balls of radius $L_{k}$, then it is $m$ - $\mathscr{L}$ oc.

Proof. One can proceed as in the proof of the previous lemma, but with the functions $f_{j}:\left(\mathbf{v}^{\prime}, \mathbf{v}^{\prime \prime}\right) \mapsto\left|\psi_{j}\left(\mathbf{v}^{\prime}\right) \psi_{j}\left(\mathbf{v}^{\prime \prime}\right)\right|$, where $\psi_{j}, j \in\left[1,\left|\mathbf{B}_{L_{k+1}}(\mathbf{u})\right|\right]$, are normalized eigenfunctions of operator $\mathbf{H}_{\mathbf{B}_{L_{k+1}}(\mathbf{u})}$. Notice that the $E_{j}$-nonresonance condition for $\mathbf{B}_{L_{k+1}}(\mathbf{u})$ is not required here, since one has $\left\|\boldsymbol{\psi}_{j}\right\|=1$, so the function $f_{j}$ is globally bounded by 1 . Let $\mathbf{x}, \mathbf{y} \in \mathbf{B}_{L_{k+1}}(\mathbf{u})$ and assume $\rho(\mathbf{x}, \mathbf{y})=R \geq L_{k}^{1+\varrho}$.

Arguing as in the previous lemma and using the dominated decay of the function $f_{j}(\cdot, \cdot)$ (cf. Lemma 10), we obtain, for any pair of points with $\rho\left(\mathbf{y}^{\prime}, \mathbf{y}^{\prime \prime}\right) \geq L_{k}^{1+\varrho}$,

$$
\begin{aligned}
& -\ln \left|\psi_{j}(\mathbf{x}) \psi_{j}(\mathbf{y})\right| \\
& \geq-\ln \left[\left(\mathrm{e}^{-m\left(1+(1 / 2) L_{k}^{-\tau}\right) L_{k}}\right)^{\left(R-2 C_{N} L_{k}-2 L_{k}\right) /\left(L_{k}+1\right)}\right] .
\end{aligned}
$$

A direct comparison with the RHS of the first equation in (59) shows that the RHS of (60) is bigger, owing to the absence of 
the large factor $\mathrm{e}^{L_{k}^{\beta}}$. Therefore, it admits the same (or better) lower bound as in (59).

Below we summarize the relations between the parameters used in the above proof:

$$
\begin{aligned}
\rho(\mathbf{x}, \mathbf{y}) & \geq L_{k-1}^{1+\varrho}, \\
0 & <\tau<\rho, \\
1+\varrho & <\alpha, \\
\beta & <1-\tau .
\end{aligned}
$$

These conditions are fulfilled, for example, with

$$
\begin{aligned}
& \alpha=\frac{4}{3}, \\
& \rho=\frac{1}{6}, \\
& \tau=\frac{1}{8}, \\
& \beta=\frac{1}{2} .
\end{aligned}
$$

\section{Scaling Analysis of Eigenfunctions: Finite-Range Interaction}

\subsection{Initial Scale Estimates}

Lemma 17 (initial scale bound). For any $L_{0}>2, m \geq 1$, and $p>0$ there is $g_{0}=g_{0}\left(m, p, L_{0}\right)<\infty$ such that for all $g$ with $|g| \geq g_{0}$, any ball $\mathbf{B}_{L_{0}}(u)$, and any $E \in \mathbb{R}$

$$
\begin{gathered}
\mathbb{P}\left\{\mathbf{B}_{L_{0}}(\mathbf{u}) \text { is }(E, m)-S\right\} \leq L_{0}^{-p}, \\
\mathbb{P}\left\{\mathbf{B}_{L_{0}}(\mathbf{u}) \text { is } m-\mathcal{N} \mathscr{L} o c\right\} \leq L_{0}^{-p} .
\end{gathered}
$$

See the proof in the Appendix.

4.2. EVC Bounds for Distant Pairs of Balls. The following statement reflects the progress achieved in the area of multiparticle eigenvalue concentration (EVC) estimates since the time when manuscript [18] had appeared.

Theorem 18 (cf. [21, Theorem 4], [19, Lemma 2.4]). Let $V$ : $\mathscr{Z} \times \Omega \rightarrow \mathbb{R}$ be a random field satisfying (W3) with parameters $b, b^{\prime} \in(0,1], A^{\prime} \in(0,+\infty)$. For all $L>0$ large enough, any distant pair of balls $\mathbf{B}_{L}^{(N)}\left(\mathbf{u}^{\prime}\right), \mathbf{B}_{L}^{(N)}\left(\mathbf{u}^{\prime \prime}\right)$ the following bound holds for any $s \in(0,1]$ :

$$
\mathbb{P}\left\{\operatorname{dist}\left(\Sigma_{L, \mathbf{u}^{\prime}}, \Sigma_{L, \mathbf{u}^{\prime \prime}}\right) \leq s\right\} \leq(3 L)^{2 N} h_{L}(2 s),
$$

where $\Sigma_{L, \mathbf{u}^{\prime}}, \Sigma_{L, \mathbf{u}^{\prime \prime}}$ are the respective spectra of $\mathbf{H}_{\mathbf{B}_{L}\left(\mathbf{u}^{\prime}\right)}$ and $\mathbf{H}_{\mathbf{B}_{L}\left(\mathbf{u}^{\prime \prime}\right)}$, and

$$
h_{L}(s):=C^{\prime \prime} L^{A^{\prime \prime}} s^{b \wedge b^{\prime}}, \quad b \wedge b^{\prime} \equiv \min \left[b, b^{\prime}\right],
$$

and $A^{\prime \prime}=A^{\prime \prime}\left(A^{\prime}, N\right) \in(0,+\infty)$. Consequently, this implies the following bound for $\beta^{\prime} \in(0, \beta)$, all L large enough and any pair $\mathbf{u}^{\prime}, \mathbf{u}^{\prime \prime}$, with $\mathrm{d}\left(\mathbf{u}^{\prime}, \mathbf{u}^{\prime \prime}\right)>4 N L$ :

$$
\mathbb{P}\left\{\exists E \in \mathbb{R}: \mathbf{B}_{L}\left(\mathbf{u}^{\prime}\right), \mathbf{B}_{L}\left(\mathbf{u}^{\prime \prime}\right) \text { are } E-P R\right\} \leq \mathrm{e}^{-L^{\beta^{\prime}}} .
$$

A considerable advantage of the above EVC bound, compared to [4, Lemma 2], is that it holds for any distant pair of balls. In [4], as well as in [17], a similar bound is established only for pairs of balls sufficiently distant with respect to the so-called Hausdorff (pseudo)metric; such a bound did not cover some pairs distant with respect to the more natural symmetrized distance, like $\boldsymbol{\rho}$. As a result, one could not prove uniform decay of eigenfunction correlators in the physically relevant distance in the multiparticle configuration space. A similar difficulty was encountered in [6].

\subsection{Partially Interactive $N$-Particle Balls}

Definition 19. An $N$-particle ball $\mathbf{B}_{L}(\mathbf{u})$ is called partially interactive (PI, in short) if diam $\mathbf{u}>A_{N} L, A_{N}=4 N$, and fully interactive (FI) otherwise.

The local Hamiltonian $\mathbf{H}_{\mathbf{B}_{L}(\mathbf{u})}^{(N)}$ with a finite-range interaction in a PI ball is algebraically decomposable as follows:

$$
\mathbf{H}_{\mathbf{B}_{L}(\mathbf{u})}^{(N)}=\mathbf{H}_{\mathbf{B}_{L}\left(\mathbf{u}^{\prime}\right)}^{\left(n^{\prime}\right)} \otimes \mathbf{1}^{\left(n^{\prime \prime}\right)}+\mathbf{1}^{\left(n^{\prime}\right)} \otimes \mathbf{H}_{\mathbf{B}_{L}\left(\mathbf{u}^{\prime \prime}\right)}^{\left(n^{\prime \prime}\right)}
$$

with $n^{\prime}+n^{\prime \prime}=N, \mathbf{u}=\left(\mathbf{u}^{\prime}, \mathbf{u}^{\prime \prime}\right), \mathbf{u}^{\prime} \in \mathscr{Z}_{>}^{\mathbf{n}^{\prime}}, \mathbf{u}^{\prime \prime} \in \mathscr{Z}_{>}^{\mathbf{n}^{\prime \prime}}$, while for a fully interactive ball one cannot guarantee such a property. (In the case of a rapidly decaying interaction, the RHS is close in norm to the LHS.)

Lemma 20. (A) If $\mathbf{B}_{L}(\mathbf{x})$ is PI, then there exists a partition of $[[1, N]]$ into nonempty complementary subsets $\mathscr{J}, \mathscr{J}^{c}$ such that $\rho\left(\Pi_{\mathcal{g}} \mathbf{B}_{L}(\mathbf{x}), \Pi_{g c} \mathbf{B}_{L}(\mathbf{x})\right)>2 L$.

(B) If $\mathbf{B}_{L}(\mathbf{x}), \mathbf{B}_{L}(\mathbf{y})$ are two FI balls with $\rho(\mathbf{x}, \mathbf{y})>11 \mathrm{NL}$, then $\rho\left(\Pi \mathbf{B}_{L}(\mathbf{x}), \Pi \mathbf{B}_{L}(\mathbf{y})\right) \geq 2 L$.

Here the value $11 N$ is obtained as $2 A_{N}+3$.

See the proof in the Appendix, Section A.2.

Now we state a result on localization in PI balls for the Hamiltonians with a short-range interaction (condition (U0)). The reader may want to compare the elementary proof of Lemma 21 below with that of Lemma 3 in [4] which is substantially more complex. The simplification comes here from the fact that we rely on the $m$ - $\mathscr{L} o c$ property, that is, exponential decay of all EFs of a Hamiltonian in a given ball, and this property is nonrelated to a specific energy but covers the entire spectrum of the Hamiltonian at hand; this results in a few-line proof of Lemma 21 as well as of an important probabilistic bound (Lemma 22) stemming from it.

Lemma 21. Assume that the interaction $\mathbf{U}$ has finite range $r_{0} \in$ $[0,+\infty)(c f .(\cup 0))$. Consider a PIN-particle ball with canonical factorization

$$
\mathbf{B}_{L_{k}}^{(N)}(\mathbf{u})=\mathbf{B}_{L_{k}}^{\left(n^{\prime}\right)}\left(\mathbf{u}^{\prime}\right) \times \mathbf{B}_{L_{k}}^{\left(n^{\prime \prime}\right)}\left(\mathbf{u}^{\prime \prime}\right)
$$


If the balls $\mathbf{B}_{L_{k-1}}^{\left(n^{\prime}\right)}\left(\mathbf{u}^{\prime}\right)$ and $\mathbf{B}_{L_{k-1}}^{\left(n^{\prime \prime}\right)}\left(\mathbf{u}^{\prime \prime}\right)$ are $m$ - $\mathscr{L}$ oc, then the ball $\mathbf{B}_{L_{k}}^{(N)}(\mathbf{u})$ is also $m-\mathscr{L}$ oc.

Proof. The interaction between subsystems corresponding to $\mathbf{u}^{\prime}$ and $\mathbf{u}^{\prime \prime}$ vanishes, so $\mathbf{H}_{\mathbf{B}_{L_{k}}(\mathbf{u})}$ admits the decomposition

$$
\mathbf{H}_{\mathbf{B}_{L_{k}}(\mathbf{u})}=\mathbf{H}_{\mathbf{B}_{L_{k}}^{\left(n^{\prime}\right)}\left(\mathbf{u}^{\prime}\right)} \otimes \mathbf{1}^{\left(n^{\prime \prime}\right)}+\mathbf{1}^{\left(n^{\prime}\right)} \otimes \mathbf{H}_{\mathbf{B}_{L_{k}}^{\left(n^{\prime \prime}\right)}\left(\mathbf{u}^{\prime \prime}\right)} .
$$

Thus the eigenfunctions $\Psi_{j}$ of $\mathbf{H}_{\mathbf{B}_{L_{k}} \text { (u) }}$ can be chosen in the form $\Psi_{j}=\phi_{j^{\prime}} \otimes \psi_{j^{\prime \prime}}$, where $\phi_{j^{\prime}}$ are eigenfunctions of $\mathbf{H}_{\mathbf{B}_{L_{k}}^{\left(n^{\prime}\right)}\left(\mathbf{u}^{\prime}\right)}$ and, respectively, $\psi_{j^{\prime \prime}}$ are eigenfunctions of $\mathbf{H}_{\mathbf{B}_{L_{k}}^{\left(n^{\prime \prime}\right)}\left(\mathbf{u}^{\prime \prime}\right)}$. Now the required decay bounds on the projection kernel,

$$
\begin{aligned}
& \left|\Psi_{j}(\mathbf{x}) \Psi_{j}(\mathbf{y})\right| \\
& \quad=\left|\phi_{j^{\prime}}\left(\mathbf{x}^{\prime}\right) \phi_{j^{\prime \prime}}\left(\mathbf{x}^{\prime \prime}\right) \psi_{j^{\prime}}\left(\mathbf{y}^{\prime}\right) \psi_{j^{\prime \prime}}\left(\mathbf{y}^{\prime \prime}\right)\right|,
\end{aligned}
$$

follow directly from the respective bounds on $\phi$ and $\psi$, which are guaranteed by the $m-\mathscr{L}$ oc hypotheses on the projection balls $\mathbf{B}_{L_{k}}^{\left(n^{\prime}\right)}\left(\mathbf{u}^{\prime}\right)$ and $\mathbf{B}_{L_{k}}^{\left(n^{\prime \prime}\right)}\left(\mathbf{u}^{\prime \prime}\right)$.

Given numbers $p, \theta>0$, introduce the quantities

$$
P(n, k)=2^{N-n} p(1+\theta)^{k}, \quad 1 \leq n \leq N ; k \geq 0 .
$$

Later it will be required that $\vartheta \in(0, \sqrt{2}-1)$.

Lemma 22. Suppose that for all $n \in[1, N-1]$ and for a given $k \geq 0$ the following bound holds for any n-particle ball $\mathbf{B}_{L_{k}}^{(n)}(\mathbf{u})$ :

$$
\mathbb{P}\left\{\mathbf{B}_{L_{k}}^{(n)}(\mathbf{u}) \text { is } m-\mathscr{N} \mathscr{L} \text { oc }\right\} \leq L_{k}^{-P(n, k)}
$$

Then for any $N$-particle PI ball $\mathbf{B}_{L_{k}}^{(N)}(\mathbf{u})$ one has

$$
\mathbb{P}\left\{\mathbf{B}_{L_{k}}^{(N)}(\mathbf{u}) \text { is } m-\mathcal{N} \mathscr{L} \text { oc }\right\} \leq 2 L_{k}^{-2 P(N, k)} \leq \frac{1}{4} L_{k}^{-P(N, k)}
$$

Proof. It follows from Lemma 21 that a ball $\mathbf{B}_{L_{k}}^{(N)}(\mathbf{u})$ admitting a factorization of the form (69) is $m-\mathscr{N} \mathscr{L}$ oc only if at least one of the projection balls $\mathbf{B}_{L_{k}}^{\left(n^{\prime}\right)}\left(\mathbf{u}^{\prime}\right), \mathbf{B}_{L_{k}}^{\left(n^{\prime \prime}\right)}\left(\mathbf{u}^{\prime \prime}\right)$ is $m-\mathscr{N} \mathscr{L} o c$. Therefore, with $L_{0}$ large enough, we obtain

$$
\begin{aligned}
& \mathbb{P}\{\left.\left.\mathbf{B}_{L_{k}}^{(N)}(\mathbf{u}) \text { is } m-\mathscr{N} \mathscr{L} \circ\right\}\right\} \\
& \leq\left.\mathbb{P}\left\{\mathbf{B}_{L_{k}}^{\left(n^{\prime \prime}\right)}\left(\mathbf{u}^{\prime \prime}\right) \text { is } m-\mathcal{N} \mathscr{L} \circ\right\}\right\} \\
& \quad+\mathbb{P}\left\{\mathbf{B}_{L_{k}}^{\left(n^{\prime \prime}\right)}\left(\mathbf{u}^{\prime \prime}\right) \text { is } m-\mathcal{N} \mathscr{L} \circ c\right\} \\
& \leq L_{k}^{-P\left(n^{\prime}, k\right)}+L_{k}^{-P\left(n^{\prime \prime}, k\right)} \leq 2 L_{k}^{-2 P(N, k)}<\frac{1}{4} L_{k}^{-P(N, k)} .
\end{aligned}
$$

4.4. Fully Interactive N-Particle Balls. Owing to Lemma 22, it remains to establish localization bounds for $N$-particle FI balls $\mathbf{B}_{L_{k+1}}(\mathbf{x})$, assuming, if necessary, similar bounds: (i) For $n$-particle balls of any radius $L_{k^{\prime}}, k^{\prime} \geq 0$, with $n<$ $N$.

(ii) For $N$-particle balls of any radius $L_{k^{\prime}}$ with $k^{\prime} \leq k$.

Lemma 23 (main inductive lemma). Suppose that for a given $k \geq 0$ and all $i \in[0, k]$ the following bound holds for any $N$ particle ball $\mathbf{B}_{L_{k}}^{(N)}(\mathbf{u})$ :

$$
\mathbb{P}\left\{\mathbf{B}_{L_{i}}^{(N)}(\mathbf{u}) \text { is } m-\mathcal{N} \mathscr{L} \text { oc }\right\} \leq L_{i}^{-P(N, i)} .
$$

Then for any $N$-particle FI ball $\mathbf{B}_{L_{k+1}}^{(n)}(\mathbf{x})$ one has

$$
\mathbb{P}\left\{\mathbf{B}_{L_{k+1}}^{(N)}(\mathbf{u}) \text { is } m-\mathcal{N} \mathscr{L} \text { oc }\right\} \leq L_{k+1}^{-P(N, k+1)},
$$

provided that

$$
\begin{aligned}
& p>\frac{2 \alpha^{2}}{2-\alpha^{2}} N d, \\
& 0<3 \theta \leq \min \left\{\frac{2-\alpha^{2}}{\alpha^{2}}-\frac{2 N d}{p}, \sqrt{2}-1\right\} .
\end{aligned}
$$

Furthermore, for any pair of distant balls $\mathbf{B}_{L_{k}}^{(N)}(\mathbf{u}), \mathbf{B}_{L_{k}}^{(N)}(\mathbf{v})$, one has, for some $a, c>0$,

$$
\begin{gathered}
\mathbb{P}\left\{\exists E \in \mathbb{R}: \mathbf{B}_{L_{k}}^{(N)}(\mathbf{u}), \mathbf{B}_{L_{k}}^{(N)}(\mathbf{v}) \text { are }(E, m)-S\right\} \\
\leq L_{k}^{-P(N, k)} \equiv L_{k}^{-p(1+9)^{k}} \leq \mathrm{e}^{-a \ln ^{1+c} L_{k}} .
\end{gathered}
$$

Proof. Introduce the following events:

$$
\begin{aligned}
& \mathcal{N}_{k+1}=\left\{\mathbf{B}_{L_{k+1}}^{(N)}(\mathbf{u}) \text { is } m-\mathcal{N} \mathscr{L} \text { oc }\right\}, \\
& \mathcal{S}_{k}^{(2)} \\
& =\left\{\exists E \exists \text { distant }(E, m) \text {-S balls } \mathbf{B}_{L_{k}}^{(N)}(\mathbf{u}), \mathbf{B}_{L_{k}}^{(N)}(\mathbf{v})\right. \\
& \left.\quad \subset \mathbf{B}_{L_{k+1}}^{(N)}(\mathbf{w})\right\}, \\
& \mathscr{R}_{k}^{(2)}=\left\{\exists E \exists \text { distant } E \text {-PR balls } \mathbf{B}_{L_{k}}^{(N)}(\mathbf{u}), \mathbf{B}_{L_{k}}^{(N)}(\mathbf{v})\right. \\
& \left.\quad \subset \mathbf{B}_{L_{k+1}}^{(N)}(\mathbf{w})\right\} .
\end{aligned}
$$

By virtue of Lemma 16, we have

$$
\mathscr{N}_{k+1} \subset \delta_{k}^{(2)} \subset \mathscr{R}_{k}^{(2)} \cup\left(\mathcal{S}_{k}^{(2)} \backslash \mathscr{R}_{k}^{(2)}\right),
$$

and by Theorem $18, \mathbb{P}\left\{\mathscr{R}_{k}^{(2)}\right\} \leq \mathrm{e}^{-L_{k}^{\beta^{\prime}}}$, so it remains to assess the probability $\mathbb{P}\left\{\mathcal{S}_{k}^{(2)} \backslash \mathscr{R}_{k}^{(2)}\right\}$. Fix points $\mathbf{u}, \mathbf{v} \in \mathbf{B}_{L_{k+1}}^{(N)}(\mathbf{w})$ and introduce the event (figuring in (78))

$$
\mathcal{S}_{k}^{(2)}(\mathbf{u}, \mathbf{v})=\left\{\exists E: \mathbf{B}_{L_{k}}^{(N)}(\mathbf{u}), \mathbf{B}_{L_{k}}^{(N)}(\mathbf{v}) \operatorname{are}(E, m)-S\right\} .
$$

Within the event $\mathcal{S}_{k}^{(2)}(\mathbf{u}, \mathbf{v}) \backslash \mathscr{R}_{k}^{(2)}$, either $\mathbf{B}_{L_{k}}^{(N)}(\mathbf{u})$ or $\mathbf{B}_{L_{k}}^{(N)}(\mathbf{v})$ must be $E$-CNR; w.l.o.g., assume that $\mathbf{B}_{L_{k}}^{(N)}(\mathbf{u})$ is $E$-CNR. Since it is $(E, m)-S$, Lemma 15 implies that it must contain a 
pair of $m-\mathcal{N} \mathscr{L}$ oc balls $\mathbf{B}_{L_{k-1}}^{(N)}\left(\mathbf{y}^{\prime}\right), \mathbf{B}_{L_{k-1}}^{(N)}\left(\mathbf{y}^{\prime \prime}\right)$ with $\mathrm{d}\left(\mathbf{y}^{\prime}, \mathbf{y}^{\prime \prime}\right)>$ $(1 / 2) L_{k-1}^{1+\delta}$. Consider two possible situations:

(1) Both $\mathbf{B}_{L_{k-1}}^{(N)}\left(\mathbf{y}^{\prime}\right)$ and $\mathbf{B}_{L_{k-1}}^{(N)}\left(\mathbf{y}^{\prime \prime}\right)$ are FI.

Apply Lemma 20, assertion (B):

$$
\boldsymbol{\rho}\left(\Pi \mathbf{B}_{L_{k-1}}(\mathbf{u}), \Pi \mathbf{B}_{L_{k-1}}(\mathbf{v})\right) \geq 2 L_{k} .
$$

Using the inductive assumption (75) and the mixing condition (W2), we have, with $L_{k-1}=L_{k+1}^{1 / \alpha^{2}}$ and $L_{0}$ large enough,

$$
\begin{aligned}
\mathbb{P} & \left.\left\{\mathbf{B}_{L_{k-1}}^{(N)}\left(\mathbf{y}^{\prime}\right), \mathbf{B}_{L_{k-1}}^{(N)}\left(\mathbf{y}^{\prime \prime}\right) \text { are } m-\mathcal{N} \mathscr{L} \circ\right\}\right\} \\
& \leq 2 L_{k+1}^{-\left(2 p / \alpha^{2}\right)(1+\theta)^{k-1}} .
\end{aligned}
$$

The number of such pairs $\mathbf{y}^{\prime}, \mathbf{y}^{\prime \prime}$ is bounded by $(1 / 2)\left|\mathbf{B}_{L_{k}}^{(N)}(\mathbf{w})\right|^{2} \leq\left(3^{2 N} / 2\right) L_{k}^{2 N}$, so in this case,

$$
\mathbb{P}\left\{\delta_{k}^{(2)} \backslash \mathscr{R}_{k}^{(2)}\right\} \leq \frac{3^{2 N}}{2} L_{k+1}^{-\left(2 p / \alpha^{2}\right)(1+\theta)^{k-1}+2 N} .
$$

A straightforward calculation shows that, for $L_{0}$ large enough, the RHS is bounded by $(1 / 4) L_{k+1}^{-P(N, k+1)}$, under conditions (77). Indeed, we need the inequality

$$
\frac{2 p}{\alpha^{2}} 2^{N-N} p(1+\theta)^{k-1}-2 N d>2^{N-N} p(1+\theta)^{k+1}
$$

or, equivalently,

$$
(1+\theta)^{2}<\frac{2 p}{\alpha^{2}}-\frac{2 N d}{p(1+\theta)^{k-1}} .
$$

By construction, $\vartheta \in(0,1)$, so one has $(1+\theta)^{2}<1+3 \vartheta$, and $2 N d / p>2 N d /\left(p(1+\theta)^{k-1}\right)$. Therefore, if $p>\left(2 \alpha^{2} /(2-\right.$ $\left.\left.\alpha^{2}\right)\right) N$ and

$$
3 \vartheta \leq \frac{2-\alpha^{2}}{\alpha^{2}}-\frac{2 N}{p},
$$

then (85) is also satisfied, so that

$$
\begin{aligned}
\mathbb{P}\left\{\mathcal{S}_{k}^{(2)}\right\} & \leq \mathbb{P}\left\{\mathscr{R}_{k}^{(2)}\right\}+\mathbb{P}\left\{\mathcal{S}_{k}^{(2)} \backslash \mathscr{R}_{k}^{(2)}\right\} \\
& \leq \mathrm{e}^{-L_{k}^{\beta^{\prime}}}+\frac{1}{4} L_{k+1}^{-P(N, k+1)}<\frac{1}{2} L_{k+1}^{-P(N, k+1)},
\end{aligned}
$$

yielding assertion (76) in case (1).

(2) Either $\mathbf{B}_{L_{k-1}}^{(N)}\left(\mathbf{y}^{\prime}\right)$ or $\mathbf{B}_{L_{k-1}}^{(N)}\left(\mathbf{y}^{\prime \prime}\right)$ is PI. Then by Lemma 22,

$$
\begin{aligned}
\mathbb{P} & \left\{\mathbf{B}_{L_{k-1}}^{(N)}\left(\mathbf{y}^{\prime}\right), \mathbf{B}_{L_{k-1}}^{(N)}\left(\mathbf{y}^{\prime \prime}\right) \text { is } m-\mathscr{N} \mathscr{L} \circ c\right\} \\
& \leq \min \left(\mathbb{P}\left\{\mathbf{B}_{L_{k-1}}^{(N)}\left(\mathbf{y}^{\prime}\right) \text { is } m-\mathscr{N} \mathscr{L} \circ c\right\},\right. \\
& \left.\mathbb{P}\left\{\mathbf{B}_{L_{k-1}}^{(N)}\left(\mathbf{y}^{\prime \prime}\right) \text { is } m-\mathscr{N} \mathscr{L} \circ c\right\}\right) \leq L_{k-1}^{-2 p(1+\theta)^{k-1}} \\
< & L_{k+1}^{-p(1+\theta)^{k+1}},
\end{aligned}
$$

provided that $(1+\theta)^{2}<2$; that is, $\theta<\sqrt{2}-1$.
Finally, a straightforward calculation shows that for some $a, c>0$ one has the inequality

$$
L_{k}^{-P(N, k)} \equiv L_{k}^{-p(1+9)^{k}} \leq \mathrm{e}^{-a \ln ^{1+c} L_{k}} .
$$

This completes the proof.

Applying Lemmas 17 and 22, we come by induction to the following result.

Theorem 24 (localization at any scale). Assume that $|g|$ is large enough, so that (63) hold true with $p>\left(2 \alpha^{2} /\left(2-\alpha^{2}\right)\right) N d$. Then for any $k \geq 0$ and any $\mathbf{u} \in \mathbb{Z}^{N d}$

$$
\mathbb{P}\left\{\mathbf{B}_{L_{k}}^{(N)}(\mathbf{u}) \text { is } m-\mathcal{N} \mathscr{L} \text { oc }\right\} \leq \frac{1}{4} L_{k}^{-p(1+\theta)^{k}}
$$

with $\theta>0$ satisfying (77). In other words, with probability $\geq 1-$ $(1 / 4) L_{k}^{-p(1+\theta)^{k}}$, for every eigenfunction $\Psi_{j}$ of operator $\mathbf{H}_{\mathbf{B}_{L_{k}}^{(N)}(\mathbf{u})}$ and for all $\mathbf{x}, \mathbf{y} \in \mathbf{B}_{L_{k}}^{(N)}(\mathbf{u})$ such that $\|\mathbf{x}-\mathbf{y}\| \geq L_{k}^{(1+\delta+\rho) / \alpha}$ one has

$$
\left|\Psi_{j}(\mathbf{x}) \Psi_{j}(\mathbf{y})\right| \leq \mathrm{e}^{-\gamma\left(m, L_{k}\right) L_{k}}<\mathrm{e}^{-m L_{k}} .
$$

Theorem 24 marks the end of the Direct Scaling Analysis of localized eigenfunctions in arbitrarily large finite balls.

\section{Exponential Decay of Eigenfunctions}

The DSA provides exponential decay bounds on the eigenbases in finite balls, not just on the Green functions, which shows that quantum transport in an arbitrarily large finite system over distances comparable with the size of the system is strongly inhibited. The proof of exponential decay of EFs in an infinitely extended configuration space requires an additional argument, originally proposed by Fröhlich et al. [9] and reformulated by von Dreifus and Klein [10]. More than 20 years after [10], it is merely a simple exercise.

Theorem 25. For $\mathbb{P}$-a.e. $\omega \in \Omega$ every normalized eigenfunction $\Psi$ of operator $\mathbf{H}(\omega)$ satisfies the following bound: for some $R(\omega), \widehat{\mathbf{x}}(\omega)$, and all $\mathbf{y}$ with $\|\mathbf{y}\| \geq R(\omega)$

$$
|\Psi(\mathbf{y})| \leq \mathrm{e}^{-m\|\mathbf{y}\|} .
$$

Proof. Pick an arbitrary vertex $\mathbf{z} \in \mathscr{Z}_{>}^{\mathbf{N}}$. By the BorelCantelli lemma combined with the probabilistic bound from Lemma 23, there is a subset $\Omega^{\prime} \subset \Omega$ with $\mathbb{P}\left\{\Omega^{\prime}\right\}=1$ such that for any $\omega \in \Omega^{\prime}$ and some $k_{0}(\omega)$, all $k \geq k_{0}$, and any $E \in \mathbb{R}$ there is no pair of $L_{k}^{1+\delta}$-distant $(E, m)$-S balls $\mathbf{B}_{L_{k}}(\mathbf{x}), \mathbf{B}_{L_{k}}(\mathbf{y}) \subset$ $\mathbf{B}_{L_{k+2}}(\mathbf{z})$. Fix $\omega \in \Omega^{\prime}$.

Let $\Psi$ be a normalized eigenfunction of $\mathbf{H}(\omega)$ with eigenvalue $\lambda$. Since $\|\Psi\|_{2} \leq 1$ implies $\|\Psi\|_{\infty} \leq 1$, there is a point $\widehat{\mathbf{x}}$ such that $\|\Psi\|_{\infty}=|\Psi(\widehat{\mathbf{x}})|$. If $\widehat{x}_{n} \in \mathbf{B}_{L_{k-1}}(0)$, then $\mathbf{B}_{L_{k}}(0)$ must be $(\lambda, m)$-S; otherwise the $(\lambda, m)$-NS property would lead to a contradiction:

$$
\|\Psi\|_{\infty}=|\Psi(\widehat{\mathbf{x}})| \leq \mathrm{e}^{-m L_{k}}\|\Psi\|_{\infty}<\|\Psi\|_{\infty} .
$$


Thus any ball $\mathbf{B}_{L_{k}}(\mathbf{y}) \subset \mathbf{B}_{L_{k+2}}(\mathbf{z})$ with $\rho(\mathbf{y}, \mathbf{z}) \in\left[L_{k+1}, L_{k+2}\right)$ (hence, with $\left.\rho(\mathbf{y}, \mathbf{z}) \geq L_{k}^{\alpha}>2 L_{k}^{1+\delta}\right)$ is $(\lambda, m)$-NS. Note that the function $\mathbf{x} \mapsto\left|\Psi_{n}(\mathbf{x})\right|$ is $\left(L_{k}, q\right)$-dominated in $\mathbf{B}_{R}(\mathbf{y})$, with $R=\rho(\mathbf{y}, \mathbf{z})-2 L_{k}^{1+\delta}-1>(1 / 2) L_{k}^{1+2 \delta}$ and $q=\mathrm{e}^{-\gamma\left(m, L_{k}\right) L_{k}}$. Recall that $\tau=\delta / 4$ (cf. (62)). By Lemma 6, for $L_{k}$ large enough,

$$
\begin{aligned}
-\frac{\ln |\Psi(\mathbf{y})|}{\rho(\mathbf{y}, \mathbf{z})} & \geq m\left(1+L_{k}^{-\tau}\right)\left(1-\frac{2 L_{k}+1}{\rho(\mathbf{y}, \mathbf{z})}\right) \\
& \geq m\left(1+\frac{1}{2} L_{k}^{-\tau}\right)>m,
\end{aligned}
$$

yielding assertion (93).

\section{EF Correlators and Dynamical Localization}

6.1. Bounds on the EFC in Finite Volumes. We use here a finite-volume variant of a "soft" argument proposed by Germinet and Klein in [11] and adapted in our earlier papers to finite-volume Hamiltonians, both single-particle (cf. [14, Memma 9]) and $N$-particle (cf. [19, Lemma 3.8]). Working with finite volumes allows us to avoid a functional-analytic complement regarding the weighted Hilbert-Schmidt norms of spectral projections of operators $\mathbf{H}(\omega)$ in the entire graph $\mathscr{Z}_{>}^{\mathbf{N}}$ and replace it with a simple application of Bessel's inequality.

Denote by $\mathscr{B}_{1}(I)$ the set of all Borel functions $\phi: \mathbb{R} \rightarrow \mathbb{C}$ with supp $\phi \subset I$ and $\|\phi\|_{\infty} \leq 1$.

Proposition 26 (cf. [14, Lemma 9], [19, Lemma 3.8]). Fix an integer $L \in \mathbb{N}^{*}$ and assume that the following bound holds for a given pair of disjoint balls $\mathbf{B}_{L}(x), \mathbf{B}_{L}(y)$ and an interval $I \subseteq \mathbb{R}$ :

$$
\mathbb{P}\left\{\exists E \in I: \mathbf{B}_{L}(\mathbf{x}), \mathbf{B}_{L}(\mathbf{y}) \text { are }(E, m)-S\right\} \leq f(L),
$$

for some $f(L)$. Then for any connected subgraph $\mathbf{\Lambda}$ containing the union $\mathbf{B}_{L}(x) \cup \mathbf{B}_{L}(y)$, one has

$$
\begin{aligned}
& \mathbb{E}\left[\sup _{\phi \in \mathscr{B}_{1}(I)}\left|\left\langle\mathbf{1}_{\mathbf{x}}\left|\phi\left(\mathbf{H}_{\Lambda}(\omega)\right)\right| \mathbf{1}_{\mathbf{y}}\right\rangle\right|\right] \\
& \leq C L^{d} e^{-m L}+f(L) .
\end{aligned}
$$

Taking into account Lemma 23 establishing the probabilistic bound for the quantity $f(L)$ figuring in the RHS of (96) and (97), we come to the following corollary of Proposition 26.

Corollary 27. Under the hypotheses of Theorem 2, for any $\mathbf{x}, \mathbf{y} \in \mathscr{Z}_{>}^{\mathbf{N}}$ with $\mathrm{d}(\mathbf{x}, \mathbf{y})>2 L+1$, any connected subset $\Lambda \supset \mathbf{B}_{L}(x) \cup \mathbf{B}_{L}(y)$

$$
\mathbb{E}\left[\sup _{\phi \in \mathscr{B}_{1}(\mathbb{R})}\left|\left\langle\mathbf{1}_{\mathbf{x}}\left|\phi\left(\mathbf{H}_{\Lambda}(\omega)\right)\right| \mathbf{1}_{\mathbf{y}}\right\rangle\right|\right] \leq C e^{-a \ln ^{1+c} \boldsymbol{\rho}(\mathbf{x}, \mathbf{y})} .
$$

6.2. Dynamical Localization in an Infinite Graph. Here we follow the same path as in earlier works by Aizenman et al. (cf., e.g., $[13,23]$ ).
Recall that if the random potential $V$ is a.s. bounded, the norm of $\mathbf{H}(\omega)$ is a.s. bounded by a constant, so its spectrum is contained in some nonrandom bounded interval $I$. Given an interval $I \subset \mathbb{R}$, denote by $\mathscr{C}_{1}(I)$ the set of all continuous functions $\phi$ on $\mathbb{R}$ with supp $\phi \subset I$ and $\|\phi\|_{\infty} \leq 1$.

Theorem 28. Consider the Hamiltonian $\mathbf{H}(\omega)$ of form (1) with a bounded random potential satisfying assumptions (W1)-(W3) and the interaction potential satisfying one of the assumptions (U0) and (U1). There is $g_{0}<+\infty$ such that if $|g| \geq g_{0}$, then the following property holds true.

For any bounded interval $I \subset \mathbb{R}$ containing the a.s. spectrum of $\mathbf{H}(\omega)$ and for all $\mathbf{x}, \mathbf{y} \in \mathscr{Z}_{>}^{\mathbf{N}}, \mathbf{x} \neq \mathbf{y}$, and with the same $c, a>0$ as in (98),

$$
\mathbb{E}\left[\sup _{\phi \in \mathscr{C}_{1}(I)}\left|\left\langle\mathbf{1}_{\mathbf{x}}|\phi(\mathbf{H}(\omega))| \mathbf{1}_{\mathbf{y}}\right\rangle\right|\right] \leq C \mathrm{e}^{-a \ln ^{1+c} \boldsymbol{\rho}(\mathbf{x}, \mathbf{y})} .
$$

Proof. We prove the claim under assumption (U0), using Corollary (97) established under this assumption. Similarly, case (U1) will follow from the analogous results proved in Section 7 for the infinite-range interactions.

Since both $V$ are $\mathbf{U}$ are bounded, the norm of the random operator $\mathbf{H}(\omega)$ is a.s. bounded by a nonrandom constant; hence there exists a nonrandom bounded interval $I \subset \mathbb{R}$ containing a.s. the spectrum $\Sigma(\mathbf{H}(\omega))$. For any ball $\mathbf{B}$ and any points $\mathbf{x}, \mathbf{y} \in \mathbf{B}$ introduce a spectral measure $\mu_{\mathbf{B}, \omega}^{\mathbf{x}, \mathbf{y}}$ uniquely defined by

$$
\begin{aligned}
& \int \phi(\lambda) d \mu_{\mathbf{B}, \omega}^{\mathbf{x}, \mathbf{y}}(\lambda) \\
& =\left\langle\mathbf{1}_{\mathbf{x}}\left|\phi\left(\mathbf{H}_{\mathbf{B}}(\omega)\right) \Pi_{I}\left(\mathbf{H}_{\mathbf{B}}(\omega)\right)\right| \mathbf{1}_{\mathbf{y}}\right\rangle,
\end{aligned}
$$

where $\phi$ is an arbitrary bounded continuous function with supp $\phi \subset I$ and similar spectral measures $\mu_{\omega}^{\mathbf{x}, \mathbf{y}}$ for the operator $\mathbf{H}(\omega)$ on the entire graph $\mathscr{Z}_{>}^{\mathbf{N}}$. If $\left\{\mathbf{B}_{L_{k}}\right\}$ is a growing sequence of balls, then $\mu_{\mathbf{B}_{L_{k}}, \omega}^{\mathbf{x}, \mathbf{y}}$ converge vaguely to $\mu_{\omega}^{\mathbf{x}, \mathbf{y}}$ as $k \rightarrow \infty$. Note that this fact holds true in a much more general context of unbounded operators: by a well-known result (cf. [24]), for the strong resolvent convergence of operators $\mathbf{H}_{n} \rightarrow \mathbf{H}$, with a common core $\mathscr{D}$, it suffices that $\mathbf{H}_{n} \phi \rightarrow \mathbf{H} \phi$ for any element $\phi \in \mathscr{D}$; in turn, this implies the vague convergence of the spectral measures $\left\langle\boldsymbol{\varphi}, \phi\left(\mathbf{H}_{n}\right) \psi\right\rangle$. In our case, we can choose as a core the subspace of all compactly supported functions, and on such functions, the operators $\mathbf{H}_{n}$ converge by stabilization.

So, by Fatou's lemma on convergent measures, for any measurable set $\mathscr{E} \subset \mathbb{R}$ and any growing sequence of balls $\mathbf{B}_{L_{k}}$,

$$
\mathbb{E}\left[\left|\mu_{\omega}^{\mathbf{x}, \mathbf{y}}\right|(\mathscr{E})\right] \leq \liminf _{k \rightarrow \infty} \mathbb{E}\left[\left|\mu_{\mathbf{B}_{L_{k}}}^{\mathbf{x}, \mathbf{y}}\right|(\mathscr{E})\right] .
$$

Therefore, the uniform bounds in finite volumes $\mathbf{B}_{L_{k}}$, established in Corollary 27, remain valid in the entire infinite graph.

Now assertion (B) of the main Theorem 2 for finite-range interactions follows by taking continuous functions of the form

$$
\phi_{t}: \lambda \longmapsto f_{I}(\lambda) \mathrm{e}^{\mathrm{i} t \lambda}, \quad t \in \mathbb{R},
$$


where $f_{I}: \mathbb{R} \rightarrow[0,1]$ is an arbitrary continuous function interpolating between 1 on an interval $I=[-R, R]$ containing a.s. the spectrum of $\mathbf{H}(\omega)$ and 0 on $\mathbb{R} \backslash[-R-1, R+1]$.

Note also that, arguing as in [23, Sect. 2.5], one can extend bound (99) to the compactly supported bounded Borel functions $\phi$.

\section{Adaptation to Infinite-Range Interactions}

In this section, we need to adapt a certain number of definitions and statements to rapidly decaying, infinite-range interactions satisfying assumption (U1). In particular,

(i) given a number $\delta \in(0,1 / 14)$, figuring in (U1), we set now

$$
\begin{aligned}
& \varrho=2 \delta, \\
& \alpha=1+4 \delta, \\
& \tau=\frac{\delta}{2}
\end{aligned}
$$

and observe that $\alpha^{2}<2, \varrho-\delta>\tau$;

(ii) we replace the decay exponent $\gamma(m, L)=m\left(1+L^{-\tau}\right)$, figuring in Definition 9 (cf. (48)), by

$$
\gamma(m, L, n):=m\left(1+L^{-\tau}\right)^{N-n+1} \text {. }
$$

Definition 29. A pair of balls $\mathbf{B}_{L}(\mathbf{x}), \mathbf{B}_{L}(\mathbf{y})$ is called distant iff $\rho(\mathbf{x}, \mathbf{y}) \geq C_{N} L^{1+\delta}$, with $C_{N}=4 N$.

Definition 30. An $N$-particle ball $\mathbf{B}_{L}(\mathbf{x})$ is called partially interactive (PI) if diam $\mathbf{u}>2 L^{1+\delta}$ and fully interactive (FI) otherwise.

Lemma 31. (A) If $\mathbf{B}_{L}(\mathbf{x})$ is PI, then there exists a partition of $[[1, N]]$ into nonempty complementary subsets $\mathscr{J}, \mathscr{J}^{c}$ such that $\rho\left(\Pi_{\mathcal{g}} \mathbf{B}_{L}(\mathbf{x}), \Pi_{\mathcal{g} c} \mathbf{B}_{L}(\mathbf{x})\right)>L^{1+\delta}$. Consequently, $\mathbf{B}_{L}^{(N)}(\mathbf{x})$ admits a factorization

$$
\mathbf{B}_{L}^{(N)}(\mathbf{x})=\mathbf{B}_{L}^{\left(n^{\prime}\right)}\left(\mathbf{x}^{\prime}\right) \times \mathbf{B}_{L}^{\left(n^{\prime \prime}\right)}\left(\mathbf{x}^{\prime \prime}\right)
$$

with $\operatorname{dist}\left(\mathbf{x}^{\prime}, \mathbf{x}^{\prime \prime}\right)>2 L^{1+\delta}$.

(B) If $\mathbf{B}_{L}(\mathbf{x}), \mathbf{B}_{L}(\mathbf{y})$ are two FI balls with $\rho(\mathbf{x}, \mathbf{y})>11 N L$, then $\rho\left(\Pi \mathbf{B}_{L}(\mathbf{x}), \Pi \mathbf{B}_{L}(\mathbf{y})\right) \geq L^{1+\delta}$.

The proof repeats verbatim that of Lemma 20 in Section A.2, but with $L$ replaced by $L^{1+\delta}$.

The most important technical modification is required for Lemma 21, the proof of which was very elementary, due to the use of eigenfunctions.

Next, introduce the following finite-range approximations $\mathbf{U}_{R}, R \geq r_{0}$, of a given interaction $\mathbf{U}$ generated by 2 -body interaction potentials $U^{(2)}(r)$ :

$$
U_{R}^{(2)}(r)=\mathbf{1}_{r \leq R} U^{(2)}(r) .
$$

Then for any configuration $\mathbf{x}=\left(\mathbf{x}^{\prime}, \mathbf{x}^{\prime \prime}\right) \in \mathscr{Z}_{>}^{\mathbf{n}^{\prime}} \times \mathscr{Z}_{>}^{\mathbf{n}^{\prime \prime}}$ with $\boldsymbol{\rho}\left(\mathbf{x}^{\prime}, \mathbf{x}^{\prime \prime}\right)>R$, the truncated interaction decomposes as follows: $\mathbf{U}_{R}(\mathbf{x})=\mathbf{U}_{R}\left(\mathbf{x}^{\prime}\right)+\mathbf{U}_{R}\left(\mathbf{x}^{\prime \prime}\right)$. As a result, for any factorized subset of the form $\mathbf{B}=\mathbf{B}_{L^{\prime}}\left(\mathbf{x}^{\prime}\right) \times \mathbf{B}_{L^{\prime \prime}}\left(\mathbf{x}^{\prime \prime}\right)$ with $\boldsymbol{\rho}\left(\mathbf{B}_{L^{\prime}}\left(\mathbf{x}^{\prime}\right), \mathbf{B}_{L^{\prime \prime}}\left(\mathbf{x}^{\prime \prime}\right)\right)>R$, the truncated Hamiltonian $\mathbf{H}_{\mathbf{B}}^{(R)}$ admits the algebraic decomposition

$$
\mathbf{H}_{\mathbf{B}}^{(R)}=\mathbf{H}_{\mathbf{B}_{L^{\prime}}\left(\mathbf{x}^{\prime}\right)}^{(R)} \otimes \mathbf{1}^{\left(n^{\prime \prime}\right)}+\mathbf{1}^{\left(n^{\prime}\right)} \otimes \mathbf{H}_{\mathbf{B}_{L^{\prime \prime}}\left(\mathbf{x}^{\prime \prime}\right)}^{(R)}
$$

Next, we fix an arbitrary $m \geq 1$ and modify the definition of a PI ball:

$$
\mathbf{B}_{L}^{(N)}(\mathbf{x}) \text { is called PI iff } \operatorname{diam}(\mathbf{x})>C_{N}=4 m N / \widetilde{m} .
$$

Therefore, a PI ball admits a factorization $\mathbf{B}_{L}^{(N)}(\mathbf{x})=$ $\mathbf{B}_{L^{\prime}}\left(\mathbf{x}^{\prime}\right) \times \mathbf{B}_{L^{\prime \prime}}\left(\mathbf{x}^{\prime \prime}\right)$ with $\operatorname{dist}\left(\mathbf{x}^{\prime}, \mathbf{x}^{\prime \prime}\right)>\left(C_{N} / 2 N\right) L=2 m L / \widetilde{m}$, so that, with $R_{k}=\left(C_{N} / 2 N\right) L_{k}$, one has

$$
\epsilon\left(R_{k}\right)<\mathrm{e}^{-2 m L_{k}}<\frac{1}{2} \mathrm{e}^{-L_{k}^{\beta}} .
$$

Lemma 32. Assume that the interaction $\mathbf{U}$ satisfies condition (U1). Fix an energy E. Let an $N$-particle ball $\mathbf{B}_{L_{k}}^{(N)}(\mathbf{u})=$ $\mathbf{B}_{L_{k}}^{\left(n^{\prime}\right)}\left(\mathbf{u}_{\mathcal{g}}\right) \times \mathbf{B}_{L_{k}}^{\left(n^{\prime \prime}\right)}\left(\mathbf{u}_{g^{c}}\right)$ and let a sample of the random potential $V(\cdot ; \omega)$ be such that

(a) $\operatorname{dist}\left(\mathbf{x}^{\prime}, \mathbf{x}^{\prime \prime}\right)>R_{k}:=\left(C_{N} / 2 N\right) L_{k}$;

(b) $\mathbf{B}_{L_{k}}^{\left(n^{\prime}\right)}\left(\mathbf{u}^{\prime}\right)$ and $\mathbf{B}_{L_{k}}^{\left(n^{\prime \prime}\right)}\left(\mathbf{u}^{\prime \prime}\right)$ are $m-\mathscr{L}$ oc;

(c) $\mathbf{B}_{L_{k}}^{(N)}(\mathbf{u})$ is E-NR.

Then the ball $\mathbf{B}_{L_{k}}^{(N)}(\mathbf{u})$ is $(E, m)-N S$.

Proof. Set $R_{k}=\left(C_{N} / 2 N\right) L_{k}$ and define $\mathbf{U}^{\left(R_{k}\right)}$ and $\mathbf{H}^{\left(R_{k}\right)}$ as above. Denote

$$
\begin{aligned}
\mathbf{H}_{\mathbf{u}, k}^{\left(R_{k}, N\right)} & =\mathbf{H}_{\mathbf{B}_{L_{k}}^{(N)}(\mathbf{u})}^{\left(R_{k}\right)}, \\
\mathbf{G}(E) & =\left(\mathbf{H}_{\mathbf{B}_{L_{k}}^{(N)}(\mathbf{u})}-E\right)^{-1},
\end{aligned}
$$

and $\mathbf{G}^{\left(R_{k}\right)}(E)=\left(\mathbf{H}_{\mathbf{u}, k}^{\left(R_{k}, N\right)}-E\right)^{-1}$. The operator $\mathbf{H}_{\mathbf{u}, k}^{\left(R_{k}, N\right)}$ admits the decomposition

$$
\mathbf{H}_{\mathbf{u}, k}^{\left(R_{k}, N\right)}=\mathbf{H}_{\mathbf{B}_{L_{k}}^{\left(n^{\prime}\right)}\left(\mathbf{u}^{\prime}\right)} \otimes \mathbf{1}^{\left(n^{\prime \prime}\right)}+\mathbf{1}^{\left(n^{\prime}\right)} \otimes \mathbf{H}_{\mathbf{B}_{L_{k}}^{\left(n^{\prime}\right)}\left(\mathbf{x}^{\prime \prime}\right)},
$$

with $\mathbf{u}=\left(\mathbf{u}^{\prime}, \mathbf{u}^{\prime \prime}\right) \in \mathbb{Z}^{d n^{\prime}} \times \mathbb{Z}^{d n^{\prime \prime}}$; thus its eigenvalues are the sums $E_{a, b}=\lambda_{a}+\mu_{b}$, where $\left\{\lambda_{a}\right\}=\Sigma\left(\mathbf{H}_{\mathbf{B}_{L_{k}}\left(\mathbf{u}^{\prime}\right)}\right)$ is the spectrum of $\mathbf{H}_{\mathbf{B}_{L_{k}}\left(\mathbf{u}^{\prime}\right)}$ and, respectively, $\left\{\mu_{b}\right\}=\Sigma\left(\mathbf{H}_{\mathbf{B}_{L_{k}}\left(\mathbf{u}^{\prime \prime}\right)}\right)$. Eigenvectors of $\mathbf{H}_{\mathbf{u}, k}^{\left(R_{k}, N\right)}$ can be chosen in the form $\Psi_{a, b}=\phi_{a} \otimes \psi_{b}$, where $\left\{\phi_{a}\right\}$ are eigenvectors of $\mathbf{H}_{\mathbf{B}_{L_{k}}^{\left(n^{\prime}\right)}\left(\mathbf{u}^{\prime}\right)}$ and $\left\{\boldsymbol{\psi}_{b}\right\}$ are eigenvectors of $\mathbf{H}_{\mathbf{B}_{L_{k}}^{\left(n^{\prime \prime}\right)}\left(\mathbf{u}^{\prime \prime}\right)}$. Note that, for each eigenvalue $E_{a, b}=\lambda_{a}+\mu_{b}$, that is, for each pair $\left(\lambda_{a}, \mu_{b}\right)$, the nonresonance assumption 
$\left|E-\left(\lambda_{a}+\mu_{b}\right)\right| \geq \mathrm{e}^{L_{k}^{\beta}}$ reads as $\left.\mid\left(E-\lambda_{a}\right)-\mu_{b}\right) \mid \geq \mathrm{e}^{L_{k}^{\beta}}$ and also as $\left.\mid\left(E-\mu_{b}\right)-\lambda_{a}\right) \mid \geq \mathrm{e}^{L_{k}^{\beta}}$. Therefore, we can write

$$
\begin{aligned}
\mathbf{G}^{\left(R_{k}\right)}(\mathbf{u}, \mathbf{y} ; E) & \\
= & \sum_{\lambda_{a}} \sum_{\mu_{b}} \frac{\phi_{a}\left(\mathbf{u}^{\prime}\right) \phi_{a}\left(\mathbf{y}^{\prime}\right) \psi_{b}\left(\mathbf{u}^{\prime \prime}\right) \psi_{b}\left(\mathbf{y}^{\prime \prime}\right)}{\left(\lambda_{a}+\mu_{b}\right)-E} \\
= & \sum_{\lambda_{a}} \mathbf{P}_{a}^{\prime}\left(\mathbf{u}^{\prime}, \mathbf{y}^{\prime}\right) \mathbf{G}_{\mathbf{B}_{L_{k}}\left(\mathbf{u}^{\prime \prime}\right)}^{\left(R_{k^{\prime}}\right)}\left(\mathbf{u}^{\prime \prime}, \mathbf{y}^{\prime \prime} ; E-\lambda_{a}\right) \\
= & \sum_{\mu_{b}} \mathbf{P}_{b}^{\prime \prime}\left(\mathbf{u}^{\prime \prime}, \mathbf{y}^{\prime \prime}\right) \mathbf{G}_{\mathbf{B}_{L_{k}}\left(\mathbf{u}^{\prime}\right)}^{\left(R_{k}\right)}\left(\mathbf{u}^{\prime}, \mathbf{y}^{\prime} ; E-\mu_{b}\right),
\end{aligned}
$$

where the resolvents $\mathbf{G}_{\mathbf{B}_{L_{k}}\left(\mathbf{u}^{\prime}\right)}^{\left(R_{k}\right)}\left(E-\mu_{b}\right)$ and $\mathbf{G}_{\mathbf{B}_{L_{k}}\left(\mathbf{u}^{\prime \prime}\right)}^{\left(R_{k}\right)}\left(E-\lambda_{a}\right)$ are nonresonant:

$$
\begin{aligned}
\left\|\mathbf{G}_{\mathbf{B}_{L_{k}}\left(\mathbf{u}^{\prime}\right)}^{\left(R_{k}\right)}\left(E-\mu_{b}\right)\right\| & \leq \mathrm{e}^{L_{k}^{\beta}}, \\
\left\|\mathbf{G}_{\mathbf{B}_{L_{k}}\left(\mathbf{u}^{\prime \prime}\right)}^{\left(R_{k}\right)}\left(E-\lambda_{a}\right)\right\| & \leq \mathrm{e}^{L_{k}^{\beta}} .
\end{aligned}
$$

For any $\mathbf{y} \in \partial^{-} \mathbf{B}_{L_{k}}(\mathbf{u})$, either $\rho\left(\mathbf{u}^{\prime}, \mathbf{y}^{\prime}\right)=L_{k}$, in which case we infer from (b)

$$
\left|\mathbf{G}^{\left(R_{k}\right)}(\mathbf{u}, \mathbf{y} ; E)\right| \leq\left|\mathbf{B}_{L_{k}}\left(\mathbf{u}^{\prime \prime}\right)\right| \mathrm{e}^{-\gamma\left(m, L_{k}, N-1\right) L_{k}+L_{k}^{\beta}},
$$

or $\rho\left(\mathbf{u}^{\prime \prime}, \mathbf{y}^{\prime \prime}\right)=L_{k}$, and then we have

$$
\left|\mathbf{G}^{\left(R_{k}\right)}(\mathbf{u}, \mathbf{y} ; E)\right| \leq\left|\mathbf{B}_{L_{k}}\left(\mathbf{u}^{\prime}\right)\right| \mathrm{e}^{-\gamma\left(m, L_{k}, N-1\right) L_{k}+L_{k}^{\beta}}
$$

In either case, the LHS is bounded by

$$
\begin{aligned}
& \exp \left(-m\left(1+L_{k}^{-\tau}\right)^{N-(N-1)+1} L_{k}+L_{k}^{\beta}+C \ln L_{k}\right) \\
& \quad<\frac{1}{2} \mathrm{e}^{-\gamma\left(m, L_{k}, N\right)} .
\end{aligned}
$$

Next, using the second resolvent identity,

$$
\mathbf{G}=\mathbf{G}^{\left(R_{k}\right)}-\mathbf{G}^{\left(R_{k}\right)}\left(\mathbf{U}-\mathbf{U}^{\left(R_{k}\right)}\right) \mathbf{G},
$$

and the assumed $E$-NR property of the resolvents $\mathbf{G}, \mathbf{G}^{\left(R_{k}\right)}$, we conclude that

$$
\begin{aligned}
\left\|\mathbf{G}-\mathbf{G}^{\left(R_{k}\right)}\right\| & \leq\left\|\mathbf{U}-\mathbf{U}^{\left(R_{k}\right)}\right\|\left\|\mathbf{G}^{\left(R_{k}\right)}\right\|\|\mathbf{G}\| \leq \mathrm{e}^{-2 m L_{k}} \mathrm{e}^{2 L_{k}^{\beta}} \\
& \leq \frac{1}{2} \mathrm{e}^{-\gamma\left(m, L_{k}, N\right)} .
\end{aligned}
$$

Now the claim follows from the inequality

$$
\begin{aligned}
\left|\mathbf{G}(\mathbf{x}, \mathbf{y} ; E)-\mathbf{G}^{\left(R_{k}\right)}(\mathbf{x}, \mathbf{y} ; E)\right| \leq \frac{1}{2} \mathrm{e}^{-\gamma\left(m, L_{k}, N\right)}, & \\
& \forall \mathbf{x}, \mathbf{y} \in \mathbf{B}_{L_{k+1}}^{(N)}(\mathbf{u}) .
\end{aligned}
$$

Lemma 33. Let $\mathbf{B}_{L_{k+1}}(\mathbf{x}), \mathbf{B}_{L_{k+1}}(\mathbf{y})$ be two distant PI balls. Then, for $L_{0}$ large enough,

$$
\begin{aligned}
\mathbb{P} & \left\{\mathbf{B}_{L_{k+1}}(\mathbf{x}), \mathbf{B}_{L_{k+1}}(\mathbf{y}) \text { are }(E, m)-S\right\} \\
& <\frac{1}{4} L_{k+1}^{-P(N, k+1)} .
\end{aligned}
$$

Proof. Let

$$
\begin{aligned}
\mathscr{R}^{(2)} & =\left\{\exists E: \mathbf{B}_{L_{k+1}}(\mathbf{x}), \mathbf{B}_{L_{k+1}}(\mathbf{y}) \text { are } E-\mathrm{R}\right\}, \\
\mathcal{S}^{(2)} & =\left\{\mathbf{B}_{L_{k+1}}(\mathbf{x}), \mathbf{B}_{L_{k+1}}(\mathbf{y}) \text { are }(E, m)-S\right\} .
\end{aligned}
$$

Then $\mathbb{P}\left\{\mathcal{S}^{(2)}\right\} \leq \mathbb{P}\left\{\mathscr{R}^{(2)}\right\}+\mathbb{P}\left\{\mathcal{S}^{(2)} \backslash \mathscr{R}^{(2)}\right\}$, and within the event $\delta^{(2)} \backslash \mathscr{R}^{(2)}$ one of the balls $\mathbf{B}_{L_{k+1}}(\mathbf{x}), \mathbf{B}_{L_{k+1}}(\mathbf{y})$ must be $E-N R$, no matter how $E \in \mathbb{R}$ is chosen.

Next, consider the canonical factorization of the PI ball

$$
\mathbf{B}_{L_{k+1}}^{(N)}(\mathbf{x})=\mathbf{B}_{L_{k+1}}^{\left(n^{\prime}\right)}\left(\mathbf{x}^{\prime}\right) \times \mathbf{B}_{L_{k+1}}^{\left(n^{\prime}\right)}\left(\mathbf{x}^{\prime \prime}\right) .
$$

By Lemma 32, if both $\mathbf{B}_{L_{k+1}}\left(\mathbf{x}^{\prime}\right)$ and $\mathbf{B}_{L_{k+1}}\left(\mathbf{x}^{\prime \prime}\right)$ are $m$ - $\mathscr{L}$ oc and $\mathbf{B}_{L_{k+1}}(\mathbf{x})$ is $(E, m)-S$, then $\mathbf{B}_{L_{k+1}}(\mathbf{x})$ is $E$-R; hence within the complement of the event $\mathscr{R}^{(2)}$, the other ball $\mathbf{B}_{L_{k+1}}(\mathbf{y})$ must be $E-N R$. On the other hand, consider the canonical factorization of the PI ball

$$
\mathbf{B}_{L_{k+1}}^{(N)}(\mathbf{y})=\mathbf{B}_{L_{k+1}}^{\left(n^{\prime}\right)}\left(\mathbf{y}^{\prime}\right) \times \mathbf{B}_{L_{k+1}}^{\left(n^{\prime \prime}\right)}\left(\mathbf{y}^{\prime \prime}\right)
$$

If both $\mathbf{B}\left(n^{\prime}\right)_{L_{k+1}}\left(\mathbf{y}^{\prime}\right)$ and $\mathbf{B}\left(n^{\prime \prime}\right)_{L_{k+1}}\left(\mathbf{y}^{\prime \prime}\right)$ are $m$ - $\mathscr{L} O c$ and $\mathbf{B}_{L_{k+1}}^{(N)}(\mathbf{y})$ is $(E, m)-S$, then $\mathbf{B}_{L_{k+1}}^{(N)}(\mathbf{y})$ must be $E-\mathrm{R}$. Let

$$
\begin{gathered}
\mathscr{L}^{(4)}=\left\{\text { one of the balls } \mathbf{B}_{L_{k+1}}^{\left(n^{\prime}\right)}\left(\mathbf{x}^{\prime}\right), \mathbf{B}_{L_{k+1}}^{\left(n^{\prime \prime}\right)}\left(\mathbf{x}^{\prime \prime}\right),\right. \\
\left.\mathbf{B}_{L_{k+1}}^{\left(n^{\prime}\right)}\left(\mathbf{y}^{\prime}\right), \mathbf{B}_{L_{k+1}}^{\left(n^{\prime}\right)}\left(\mathbf{y}^{\prime \prime}\right) \text { is } m-\mathscr{N} \mathscr{L} \circ c\right\} .
\end{gathered}
$$

Since these four balls correspond to systems with less than $N$ particles, we can use induction in $N$ and write

$$
\mathbb{P}\left\{\mathscr{L}^{(4)}\right\} \leq 4 L_{k+1}^{-P(N-1, k+1)}=4 L_{k+1}^{-2 P(N, k+1)} .
$$

Then, as we have noticed, $\mathcal{S}^{(2)} \backslash \mathscr{L}^{(4)} \subset \mathscr{R}^{(2)}$; hence, for $L_{0}$ large enough,

$$
\begin{aligned}
\mathbb{P}\left\{\mathcal{S}^{(2)}\right\} & \leq \mathbb{P}\left\{\mathscr{R}^{(2)}\right\}+\mathbb{P}\left\{\mathscr{L}^{(4)}\right\} \\
& \leq \mathrm{e}^{-L_{k}^{\beta}}+4 L_{k+1}^{-2 P(N, k+1)}<\frac{1}{4} L_{k+1}^{-P(N, k+1)} .
\end{aligned}
$$

The statement of Lemma 15 remains unchanged, but its proof requires a minor modification.

Proof of Lemma 15 under hypothesis (U1). (A) By assumption, either $\mathbf{B}_{L_{k+1}}(\mathbf{u})$ is $E$-CNR and contains no $(E, m)-S$ ball of radius $L_{k}$ or there is a point $\mathbf{w} \in \mathbf{B}_{L_{k+1}}$ (u) such that any ball $\mathbf{B}_{L_{k}}(\mathbf{v}) \subset \mathbf{B}_{L_{k+1}}(\mathbf{u})$ with $\rho(\mathbf{w}, \mathbf{v}) \geq C_{N} L_{k}^{1+\delta}$ is $(E, m)$-NS. In the former case, such an exclusion is unnecessary, but in order to 
treat both situations with one argument, we can formally set $\mathbf{w}=\mathbf{u}$ (or any other point).

Fix points $\mathbf{x}, \mathbf{y}$ with $R:=\rho(\mathbf{x}, \mathbf{y})>L_{k}^{1+\varrho}$, where $\varrho=2 \delta$ is defined in (103). Note that for $L_{0}$ large (hence, $L_{k}$ also large), $L_{k}^{1+\varrho} \gg L_{k}^{1+\delta}$. By triangle inequality,

$$
\begin{aligned}
& \rho\left(\mathbf{x}, \mathbf{B}_{\left(C_{N}-1\right) L_{k}}(\mathbf{w})\right)+\rho\left(\mathbf{y}, \mathbf{B}_{\left(C_{N}-1\right) L_{k}}(\mathbf{w})\right) \\
& \quad \geq R-\left(2 C_{N}-2\right) L_{k}^{1+\delta} .
\end{aligned}
$$

Assume first that

$$
\begin{aligned}
& r^{\prime}:=\rho\left(\mathbf{x}, \mathbf{B}_{\left(C_{N}-1\right) L_{k}^{1+\delta}}(\mathbf{w})\right) \geq L_{k}+1, \\
& r^{\prime \prime}:=\rho\left(\mathbf{y}, \mathbf{B}_{\left(C_{N}-1\right) L_{k}^{1+\delta}}(\mathbf{w})\right) \geq L_{k}+1 .
\end{aligned}
$$

All the balls of radius $L_{k}$ both in $\mathbf{B}_{r^{\prime}}(\mathbf{x})$ and in $\mathbf{B}_{r^{\prime \prime}}(\mathbf{y})$ are automatically $(E, m)$-NS. Furthermore, $r^{\prime}+r^{\prime \prime} \geq R-2\left(C_{N}-\right.$ 1) $L_{k}^{1+\delta}-2 \geq R-2 C_{N} L_{k}^{1+\delta}$.

Consider the set $\mathbf{B}=\mathbf{B}_{r^{\prime}}(\mathbf{x}) \times \mathbf{B}_{r^{\prime \prime}}(\mathbf{y})$ and the function $f: \mathbf{B} \rightarrow \mathbb{C}$ defined by $f\left(\mathbf{x}^{\prime}, \mathbf{x}^{\prime \prime}\right)=\mathbf{G}_{\mathbf{B}_{L_{k+1}}}\left(\mathbf{x}^{\prime}, \mathbf{x}^{\prime \prime} ; E\right)$. Since $E$ is not a pole of the resolvent $\mathbf{G}_{\mathbf{B}_{L_{k+1}}(\mathbf{u})}(\cdot)$, it is well defined (hence, bounded, on a finite set). By Lemma 10, $f$ is $\left(L_{k}, q\right)$-dominated both in $\mathbf{x}^{\prime}$ and in $\mathbf{x}^{\prime \prime}$, with $q \leq \mathrm{e}^{-\gamma\left(m, L_{k}, n\right)}$. Therefore, one can write, with the convention $-\ln 0=+\infty$, using Lemma 7 and setting for brevity $J:=M-n+1$,

$$
\begin{aligned}
& -\ln f(\mathbf{u}, \mathbf{y}) \\
& \geq-\ln \left[\left(\mathrm{e}^{-m\left(1+(1 / 2) L_{k}^{-\tau}\right)^{J} L_{k}}\right)^{\left(R-2 C_{N} L_{k}^{1+\delta}-2 L_{k}\right) /\left(L_{k}+1\right)}\right. \\
& \left.\cdot \mathrm{e}^{L_{k+1}^{\beta}}\right]=m\left(1+\frac{1}{2} L_{k}^{-\tau}\right)^{J} \frac{L_{k}}{L_{k}+1} R\left(1-\frac{3 C_{N}}{R}\right. \\
& \left.\cdot L_{k}\right)-L_{k+1}^{\beta}=m R\left[\left(1+\frac{1}{2} L_{k}^{-\tau}\right)^{J}\left(1-L_{k}^{-1}\right)\right. \\
& \left.\cdot\left(1-\frac{3 C_{N} L_{k}}{L_{k}^{1+\varrho}}\right)-\frac{L_{k+1}^{\beta}}{m R}\right]=m R\left[\left(1+\frac{1}{2} L_{k}^{-\tau}\right)^{J}\right. \\
& \left.\cdot\left(1-L_{k}^{-1}\right)\left(1-3 C_{N} L_{k}^{-\varrho}\right)-\frac{L_{k+1}^{\beta}}{m L_{k}^{1+\varrho}}\right] \geq m\left(1+\frac{1}{4}\right. \\
& \left.\cdot L_{k}^{-\tau}\right)^{J} R \geq \gamma\left(m, L_{k+1}\right) R .
\end{aligned}
$$

If $r^{\prime}=0$ (resp., $r^{\prime \prime}=0$ ), the required bound follows from the dominated decay of the function $f\left(\mathbf{x}^{\prime}, \mathbf{x}^{\prime \prime}\right)$ in $\mathbf{x}^{\prime \prime}$ (resp., in $\mathbf{x}^{\prime}$ ).

(B) This assertion is proved in the same way as its counterpart in Section 3.3.

The rest of the scaling procedure presented in Section 4 requires no modification and applies to infinite-range interactions.

\section{Fermionic Hamiltonians on More General Graphs}

The reduction to a standard lattice Laplacian on a subset $\left\{\left(x_{1}, \ldots, x_{N}\right): x_{1}<\cdots<x_{N}\right\}$ with Dirichlet boundary conditions is no longer possible for particle systems on lattices $\mathbb{Z}^{d}$ with $d>1$. Instead, one has to work with a symmetric power of the lattice, considered as a graph. So it seems reasonable to consider a fairly general, countable connected graph $\mathscr{Z}$ satisfying the condition of polynomial growth of balls:

$$
\forall x \in \mathscr{Z} \quad \forall L \geq 1 \quad\left|B_{L}(x)\right| \leq C_{d} L^{d},
$$

where $B_{L}(x)=\left\{y \in \mathscr{Z}: \mathrm{d}_{\mathscr{Z}}(x, y) \leq L\right\}$. In particular, this gives a uniform bound on the coordination numbers, $n_{\mathscr{Z}}(x) \leq C_{d}$ (of course, this bound may be nonoptimal).

An orthonormal basis in the Hilbert space of squaresummable antisymmetric functions $\Psi: \mathscr{Z}^{N} \rightarrow \mathbb{C}$ is formed by the functions

$$
\Phi_{\mathbf{a}}=\frac{1}{\sqrt{N !}} \sum_{\pi \in \overleftarrow{S}_{N}} \bigotimes_{j=1}^{N} \mathbf{1}_{a_{\pi^{-1}(j)}},
$$

where $\mathbf{a}=\left\{a_{1}, \ldots, a_{N}\right\}$ with $\#\left\{a_{1}, \ldots, a_{N}\right\}=N$.

Further, define the graph $\left(\mathscr{Z}_{>}^{\mathbf{N}}, \mathscr{C}_{>}^{\mathbf{N}}\right)$ as follows: the vertex set is

$$
\begin{aligned}
& \mathscr{Z}_{>}^{\mathbf{N}} \\
& \quad=\left\{\mathbf{a}=\left\{a_{1}, \ldots, a_{N}\right\}: a_{j} \in \mathscr{Z}, \#\left\{a_{1}, \ldots, a_{N}\right\}=N\right\} .
\end{aligned}
$$

Two vertices $\mathbf{a}, \mathbf{b}$ form an edge iff

(i) the symmetric difference $\mathbf{a} \ominus \mathbf{b}$ has cardinality 1 ; that is, $\mathbf{a}=\left\{a_{1}, c_{2}, \ldots, c_{N}\right\}, \mathbf{b}=\left\{b_{1}, c_{2}, \ldots, c_{N}\right\}$, with $\#\left\{a_{1}, b_{1}, c_{2}, \ldots, c_{N}\right\}=N+1$;

(ii) $\mathrm{d}_{\mathscr{Z}}\left(a_{1}, b_{1}\right)=1$.

Next, define on $\mathscr{Z}_{>}^{\mathbf{N}}$ the max-distance,

$$
\rho(\mathbf{x}, \mathbf{y})=\min _{\pi \in \overleftarrow{S}_{N}} \max _{1 \leq j \leq N} \mathrm{~d}_{\mathscr{Z}}\left(x_{\pi^{-1}(j)}, y_{j}\right),
$$

and introduce the balls $\mathbf{B}_{L}(\mathbf{x})$ relative to the distance $\rho(\cdot, \cdot)$.

Now one can define the fermionic (negative) Laplacian $(-\Delta)$ on $\mathscr{Z}_{>}^{\mathrm{N}}$ and random Hamiltonians $\mathbf{H}(\omega)=-\Delta+$ $g \mathbf{V}(\mathbf{x} ; \omega)+\mathbf{U}(\mathbf{x})$, where

$$
\mathbf{U}(\mathbf{x})=\sum_{i \neq j} U\left(\mathrm{~d}\left(x_{i}, x_{j}\right)\right), \quad U: \mathbb{N} \longrightarrow \mathbb{R},
$$

and the external random potential energy,

$$
\mathbf{V}(\mathbf{x} ; \omega)=V\left(x_{1} ; \omega\right)+\cdots+V\left(x_{N} ; \omega\right),
$$

is generated by a random field $V: \mathscr{Z} \times \Omega \rightarrow \mathbb{R}$.

The method presented in Sections 3-7 applies to strongly disordered random Hamiltonians $\mathbf{H}(\omega)=-\boldsymbol{\Delta}+g \mathbf{V}(\mathbf{x} ; \omega)+$ $\mathbf{U}(\mathbf{x})$ describing fermionic systems on connected graphs $\mathscr{Z}$ 
with polynomial growth of balls; indeed, one can see that we did not use particular properties of the one-dimensional lattice $\mathscr{Z}=\mathbb{Z}$ other than polynomially bounded growth of balls.

Strongly disordered bosonic systems can be treated in a similar way; the only modification required here concerns the explicit form of the matrix elements of the Laplacian, which remains a second-order finite-difference operator.

\section{Appendix}

\section{A. Proofs of Auxiliary Statements}

A.1. Proof of Lemma 17. We start with the second assertion. The random potential energy $\mathrm{V}(\omega)$ reads as follows:

$$
\mathbf{V}\left(x_{1}, \ldots, x_{N} ; \omega\right)=\sum_{y \in \mathbf{x}} \mathbf{n}_{y} V(y ; \omega)
$$

Therefore, if $\rho(\mathbf{x}, \mathbf{y}) \neq 0$, then there exists a point $w \in \Pi \mathbf{B}_{L}(\mathbf{u})$ such that $\mathbf{n}_{w}(\mathbf{x}) \neq \mathbf{n}_{w}(\mathbf{y})$. As a result,

$$
\begin{aligned}
\mathbf{V}(\mathbf{x} ; \omega)-\mathbf{V}(\mathbf{y} ; \omega)= & \left(\mathbf{n}_{w}(\mathbf{x})-\mathbf{n}_{w}(\mathbf{y})\right) V(w ; \omega) \\
& +\sum_{v \neq w} c_{v} V(v ; \omega)
\end{aligned}
$$

where the explicit form of the integer coefficients

$$
c_{v}=\mathbf{n}_{v}(\mathbf{x})-\mathbf{n}_{v}(\mathbf{y})
$$

is irrelevant for our argument: it suffices to know that the sum in the RHS of (A.2) is measurable with respect to the sigmaalgebra $\mathfrak{F}_{\neq w}$ generated by the random variables $\{V(v ; \cdot), v \neq$ $w\}$, while

$$
\mathbf{n}_{w}(\mathbf{x})-\mathbf{n}_{w}(\mathbf{y})=: c_{w} \in \mathbb{Z} \backslash\{0\}, \text { hence }\left|c_{w}\right| \geq 1 .
$$

Therefore,

$$
\begin{aligned}
\mathbb{P} & \{|g \mathbf{V}(\mathbf{x} ; \omega)-g \mathbf{V}(\mathbf{y} ; \omega)| \leq s\} \\
& =\mathbb{E}\left[\mathbb{P}\left\{|\mathbf{V}(\mathbf{x} ; \omega)-\mathbf{V}(\mathbf{y} ; \omega)| \leq|g|^{-1} s \mid \mathfrak{F}_{\neq w}\right\}\right] \\
& =\mathbb{E}\left[\mathbb{P}\left\{\left.\left|c_{w} V(w ; \omega)\right| \mathfrak{z}(\omega)|\leq| g\right|^{-1} s \mid \mathfrak{F}_{\neq w}\right\}\right]
\end{aligned}
$$

with some $\mathfrak{F}_{\neq w}$-measurable random variable $\mathfrak{z}(\omega)$, rendered nonrandom by conditioning on $\mathfrak{F}_{\neq w}$. The event figuring in the above conditional probability has the form

$$
\begin{aligned}
\{\omega & \left.: c_{w} V(w ; \omega) \in\left[\mathfrak{z}(\omega)-|g|^{-1} s, \mathfrak{z}(\omega)+|g|^{-1} s\right]\right\} \\
& =\{\omega: V(w ; \omega) \\
& \left.\in\left[\mathfrak{z}(\omega)-\frac{s|g|^{-1}}{c_{w}}, \mathfrak{z}(\omega)+\frac{s|g|^{-1}}{c_{w}}\right]\right\} .
\end{aligned}
$$

Since $\left|c_{w}\right| \geq 1$ has length $2 s$, we conclude that

$$
\begin{aligned}
& \mathbb{P}\left\{|\mathbf{V}(\mathbf{x} ; \omega)-\mathbf{V}(\mathbf{y} ; \omega)| \leq|g|^{-1} s\right\} \\
& \leq \sup _{a \in \mathbb{R}}\left(F_{V, w}(a+2 s)-F_{V, w}(a)\right),
\end{aligned}
$$

where $F_{V, w}(\cdot)$ is the conditional PDF of the random field $V$ at $w$, given $\mathfrak{F}_{\neq w}$. Since $F_{V, w}$ is continuous, by assumption (W1), the RHS of (A.7) vanishes as $|g| \rightarrow \infty$. Therefore, the probability

$$
\mathbb{P}\left\{\exists \mathbf{x}, \mathbf{y} \in \mathbf{B}_{L}(\mathbf{u}): \mathbf{x} \neq \mathbf{y},|g \mathbf{V}(\mathbf{x})-g \mathbf{V}(\mathbf{y})| \leq s\right\}
$$

tends to 0 as $|g| \rightarrow \infty$, so with arbitrarily high probability, the spectrum of the diagonal operator $\mathbf{V}(\omega)$ in $\mathbf{B}_{L_{0}}(\mathbf{u})$ admits a positive uniform lower bound $s>0$ on all spectral spacings (differences between the eigenvalues). By taking $|g|$ large enough, all spacings for operator $g \mathbf{V}(\omega)$ can be made arbitrarily large. Eigenvectors of a continuous finitedimensional operator family $A(t)$ with simple spectrum at $t=t_{0}$ are continuous in a neighborhood of $t_{0}$. To prove the second assertion, it suffices to apply this fact to the family $A(t)=\mathbf{W}-g^{-1} t \Delta, t \in[0,1]$.

The proof of the first assertion is even simpler. Using again representation (A.1), we see that for each $\mathbf{x}$ the value of the potential energy $\mathbf{V}(\mathbf{x} ; \omega)+\mathbf{U}(\mathbf{x})$ is a linear combination (with integer coefficients) of random variables with continuous probability distribution obeying (W1). Arguing as above, one can see that, for any $E \in \mathbb{R}, s>0$, and $|g|$ large enough, with probability arbitrarily close to $1, \operatorname{dist}\left(E, \Sigma\left(\mathbf{H}_{\mathbf{B}_{L_{0}}(\mathbf{u})}\right)\right) \geq s$. By the Combes-Thomas estimate [25], this implies exponential decay of the Green functions,

$$
\left|\mathbf{G}_{\mathbf{B}_{L_{0}}(\mathbf{u})}(\mathbf{x}, \mathbf{y} ; E)\right| \leq \mathrm{e}^{-m(s) \mathrm{d}(\mathbf{x}, \mathbf{y})},
$$

with $m(s) \rightarrow \infty$ as $s \rightarrow \infty$. Since the graph distance $\mathrm{d}(\cdot, \cdot)$ on $\mathscr{Z}_{>}^{\mathbf{N}}$ dominates the max-distance $\rho(\cdot, \cdot)$, the claim follows.

\section{A.2. Proof of Lemma 20}

Proof. (A) Consider the set $\Pi B_{2 L}(\mathbf{x}) \equiv \bigcup_{j=1}^{n} B_{2 L}\left(x_{j}\right)$. If diam $\Pi \mathbf{x}>A_{N} L$, this union cannot be connected, for otherwise it would have diameter $\leq N \cdot 4 L$, but diam $\Pi B_{2 L}(\mathbf{x}) \geq$ $\operatorname{diam} \Pi \mathbf{x}>A_{N} L=4 N L$. Therefore, $\Pi B_{L^{1+\delta}}(\mathbf{x})$ can be decomposed into a disjoint union of two nonempty clusters:

$$
\left(\bigcup_{j \in \mathscr{J}} B_{2 L}\left(x_{j}\right)\right) \amalg\left(\bigcup_{i \in \mathscr{J}^{c}} B_{2 L}\left(x_{i}\right)\right),
$$

so for all $j \in \mathscr{J}, i \in \mathscr{J}^{c}$ one has

$$
\rho\left(B_{L}\left(x_{i}\right), B_{L}\left(x_{j}\right)\right)>2 L,
$$

yielding assertion (B).

(B) If $\rho(\mathbf{x}, \mathbf{y})>C_{N} L$, then for some $j_{0}, \rho\left(x_{j_{0}}, y_{j_{0}}\right)>C_{N} L$. Since both balls are FI, for all $j \in[1, n]$

$$
\begin{aligned}
& \rho\left(x_{j}, x_{j_{o}}\right) \leq \operatorname{diam} \Pi \mathbf{x} \leq A_{N} L, \\
& \rho\left(y_{j}, y_{j_{o}}\right) \leq \operatorname{diam} \Pi \mathbf{y}<A_{N} L,
\end{aligned}
$$

so by triangle inequality, for all $i, j \in[1, n]$

$$
\begin{aligned}
\rho\left(x_{i}, y_{j}\right) & \geq \rho\left(x_{j_{\circ}}, y_{j_{\circ}}\right)-\rho\left(x_{i}, x_{j_{\circ}}\right)-\rho\left(y_{j}, y_{j_{\circ}}\right) \\
& \geq C_{N} L-2 A_{N} L \geq 3 L,
\end{aligned}
$$


yielding

$$
\rho\left(\Pi B_{L}(\mathbf{x}), \Pi B_{L}(\mathbf{y})\right)>3 L-2 L=L .
$$

\section{Conflict of Interests}

The author declares that there is no conflict of interests regarding the publication of this paper.

\section{Acknowledgments}

It is a pleasure to thank Tom Spencer, Boris Shapiro, Abel Klein, and Misha Goldstein for fruitful discussions of the localization techniques, the organizers of the programs "Mathematics and Physics of Anderson Localization: 50 Years After" (2008) and "Periodic and Ergodic Spectral problems" (2015) at the Isaac Newton Institute, Cambridge, UK, and the team of the institute for their support and warm hospitality, and Shmuel Fishman, Boris Shapiro, and the Department of Physics of Technion, Israel (2009), for their support and warm hospitality.

\section{References}

[1] P. W. Anderson, "Absence of diffusion in certain random lattices," Physical Review, vol. 109, no. 5, pp. 1492-1505, 1958.

[2] D. M. Basko, I. L. Aleiner, and B. L. Altshuler, "Metal-insulator transition in a weakly interacting many-electron system with localized single-particle states," Annals of Physics, vol. 321, no. 5, pp. 1126-1205, 2006.

[3] I. V. Gornyi, A. D. Mirlin, and D. G. Polyakov, "Interacting electrons in disordered wires: anderson localization and lowT transport," Physical Review Letters, vol. 95, Article ID 206603, 2005.

[4] V. Chulaevsky and Y. Suhov, "Multi-particle Anderson localisation: induction on the number of particles," Mathematical Physics, Analysis and Geometry, vol. 12, no. 2, pp. 117-139, 2009.

[5] V. Chulaevsky, A. Boutet de Monvel, and Y. Suhov, "Dynamical localization for a multi-particle model with an alloy-type external random potential," Nonlinearity, vol. 24, no. 5, pp. 14511472, 2011.

[6] M. Aizenman and S. Warzel, "Localization bounds for multiparticle systems," Communications in Mathematical Physics, vol. 290, no. 3, pp. 903-934, 2009.

[7] M. Fauser and S. Warzel, "Multiparticle localization for disordered systems on continuous space via the fractional moment method," Reviews in Mathematical Physics, vol. 27, no. 4, Article ID 1550010, 2015.

[8] J. Fröhlich and T. Spencer, "Absence of diffusion in the Anderson tight binding model for large disorder or low energy," Communications in Mathematical Physics, vol. 88, no. 2, pp. 151184, 1983.

[9] J. Fröhlich, F. Martinelli, E. Scoppola, and T. Spencer, "Constructive proof of localization in the Anderson tight binding model," Communications in Mathematical Physics, vol. 101, no. 1, pp. 21-46, 1985.

[10] H. von Dreifus and A. Klein, "A new proof of localization in the Anderson tight binding model," Communications in Mathematical Physics, vol. 124, no. 2, pp. 285-299, 1989.
[11] F. Germinet and A. Klein, "Bootstrap multi-scale analysis and localization in random media," Communications in Mathematical Physics, vol. 222, no. 2, pp. 415-448, 2001.

[12] M. Aizenman and S. A. Molchanov, "Localization at large disorder and at extreme energies: an elementary derivation," Communications in Mathematical Physics, vol. 157, no. 2, pp. 245-278, 1993.

[13] M. Aizenman, J. H. Schenker, R. M. Friedrich, and D. Hundertmark, "Finite-volume fractional-moment criteria for Anderson localization," Communications in Mathematical Physics, vol. 224, no. 1, pp. 219-253, 2001.

[14] V. Chulaevsky, "Direct scaling analysis of localization in singleparticle quantum systems on graphs with diagonal disorder," Mathematical Physics, Analysis and Geometry, vol. 15, no. 4, pp. 361-399, 2012.

[15] Y. G. Sinai, "Anderson localization for one-dimensional difference Schrödinger operator with quasiperiodic potential," Journal of Statistical Physics, vol. 46, no. 5-6, pp. 861-909, 1987.

[16] V. Chulaevsky and Y. Suhov, "Eigenfunctions in a two-particle Anderson tight binding model," Communications in Mathematical Physics, vol. 289, no. 2, pp. 701-723, 2009.

[17] A. Klein and S. T. Nguyen, "Bootstrap multiscale analysis for the multi-particle Anderson model," Journal of Statistical Physics, vol. 151, no. 5, pp. 938-973, 2013.

[18] V. Chulaevsky, "Direct Scaling Analysis of localization in disordered systems. II. Multi-particle lattice systems," http://arxiv.org/abs/1106.2234.

[19] V. Chulaevsky and Y. Suhov, "Efficient Anderson localization boundsfor large multi-particle systems," Journal of Spectral Theory, In press, https://www.ems-ph.org/journals/forthcoming .php?jrn=jst.

[20] V. Chulaevsky, "On resonances in disordered multi-particle systems," Comptes Rendus de l'Académie des Sciences, Series I, vol. 350, pp. 81-85, 2011.

[21] V. Chulaevsky, "On the regularity of the conditional distribution of the sample mean," Markov Processes and Related Fields, vol. 21, no. 3, pp. 415-431, 2015.

[22] V. Chulaevsky and Y. Suhov, Multi-Scale Analysis for Random Quantum Systems with Interaction, Birkhäauser, Boston, Mass, USA, 2013.

[23] M. Aizenman, A. Elgart, S. Naboko, J. H. Schenker, and G. Stolz, "Moment analysis for localization in random Schrödinger operators," Inventiones Mathematicae, vol. 163, no. 2, pp. 343413, 2006.

[24] T. Kato, Perturbation Theory for Linear Operators, Springer, New York, NY, USA, 1976.

[25] J. M. Combes and L. Thomas, "Asymptotic behaviour of eigenfunctions for multiparticle Schrödinger operators," Communications in Mathematical Physics, vol. 34, pp. 251-270, 1973. 


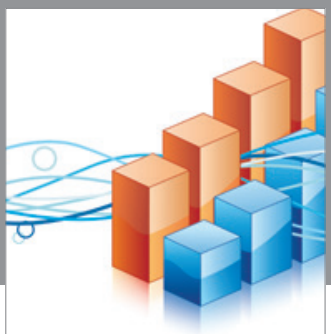

Advances in

Operations Research

vatem alat4

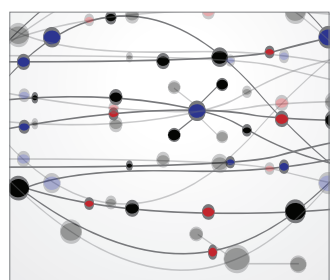

\section{The Scientific} World Journal
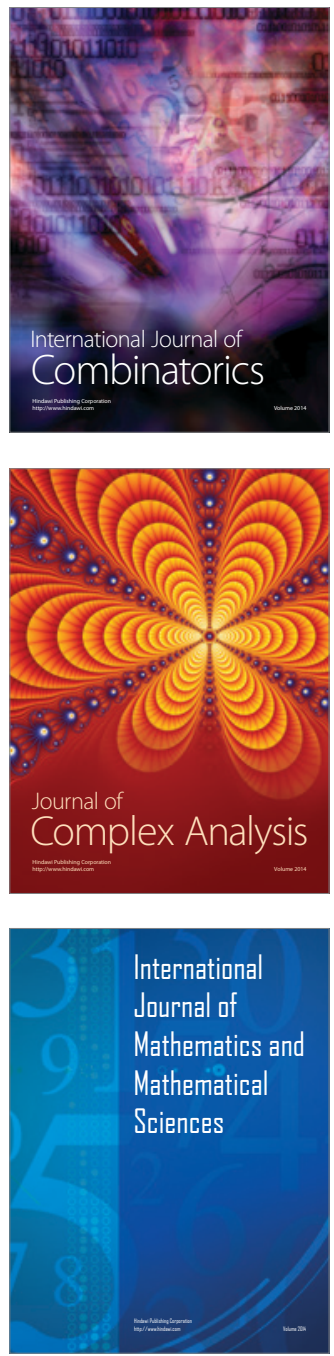
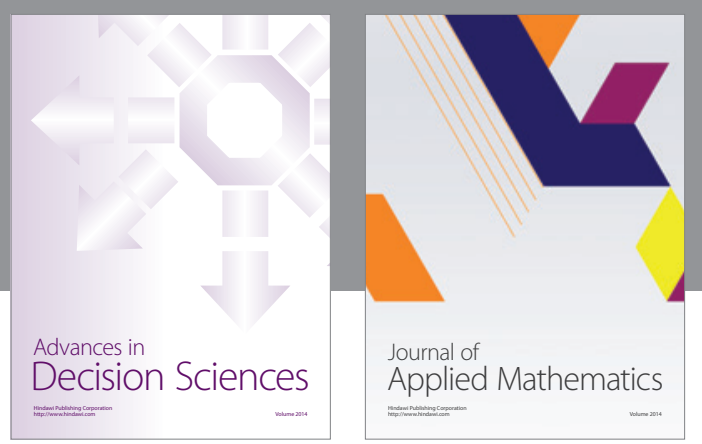

Algebra

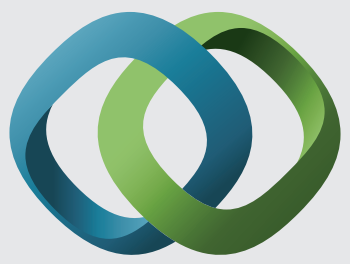

\section{Hindawi}

Submit your manuscripts at

http://www.hindawi.com
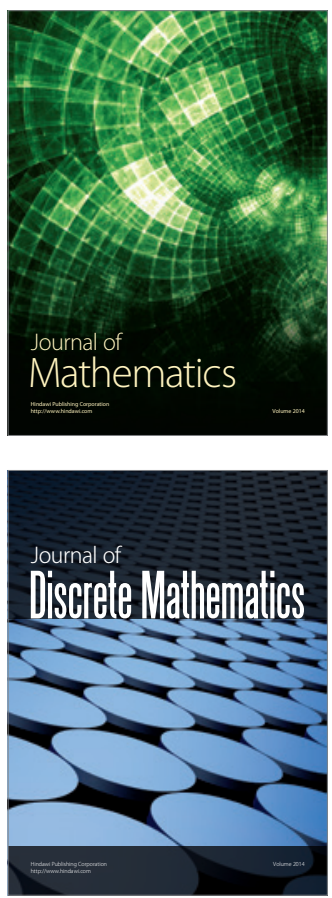

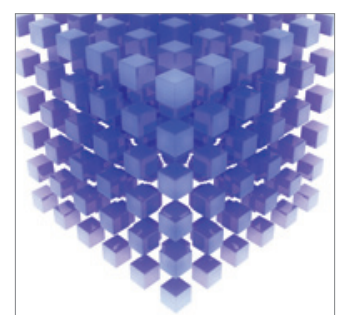

Mathematical Problems in Engineering
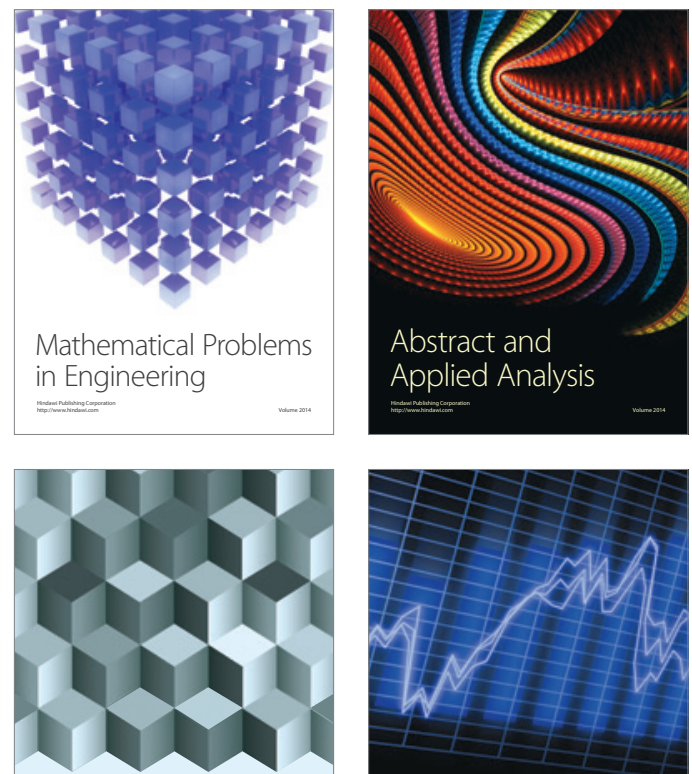

Journal of

Function Spaces

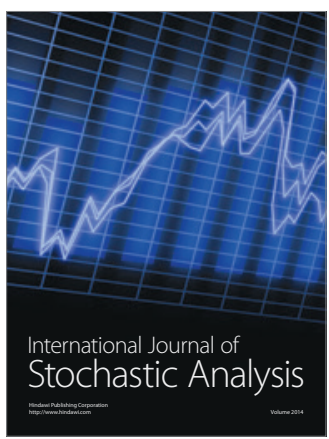

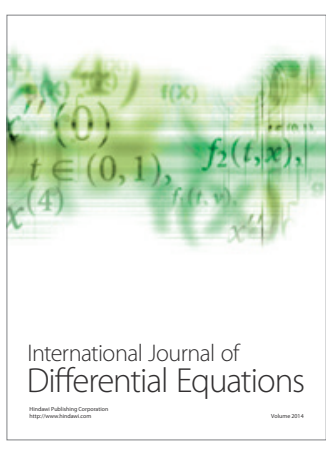
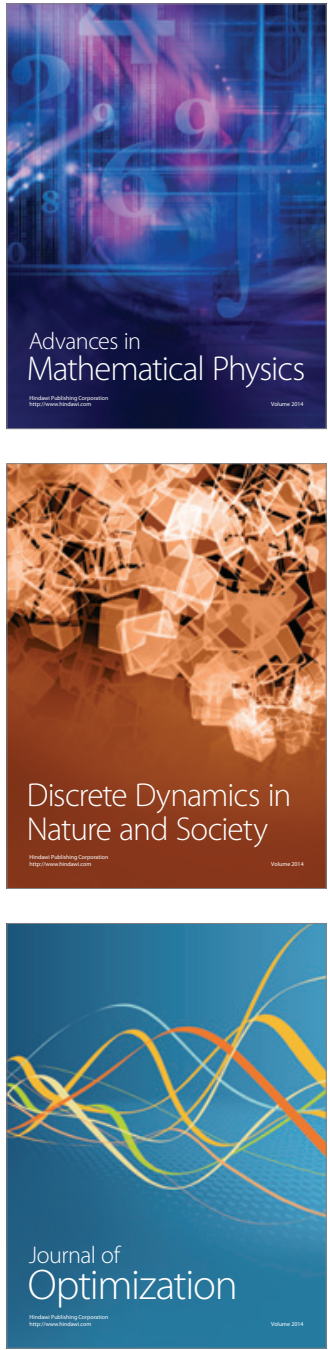